\title{
Eddy currents in a gradient coil, modeled as circular loops of strips
}

\author{
J.M.B. Kroot, S.J.L. van Eijndhoven, A.A.F. van de Ven \\ Eindhoven University of Technology \\ P.O.Box 513; 5600 MB Eindhoven, The Netherlands
}

May 17, 2005

\begin{abstract}
The z-coil of an MRI-scanner is modeled as a set of circular loops of strips, or rings, placed on one cylinder. The current in this set of thin conducting rings is driven by an external source current. The source, and all excited fields, are time harmonic. The frequency is low enough to allow for a quasi-static approximation. The rings have a thin rectangular cross-section; the thickness is so small that the current can be assumed uniformly distributed in the thickness direction. Due to induction, eddy currents occur, resulting in a so-called edge-effect. Higher frequencies cause stronger edge-effects. As a consequence, the resistance of the system increases and the self-inductance decreases. From the Maxwell equations, an integral equation for the current distribution in the rings is derived. The Galerkin method is applied, using Legendre polynomials as global basis functions, to solve this integral equation. This method shows a fast convergence, so only a very restricted number of basis functions is needed. The general method is worked out for $N(N \geq 1)$ rings, and explicit results are presented for $N=1, N=2$ and $N=24$.
\end{abstract}

\section{Introduction}

Magnetic Resonance Imaging (MRI) is a revolutionary imaging technique that plays an important role in the medical community. It provides images of cross-sections of a body without ionizing radiation, and a lot of information is obtained because MR signals are sensitive for several tissue parameters. Its excellent soft tissue contrast makes MRI the ideal technique for viewing the brain, the heart, and cardiac function, as well as the muscular, skeletal and the abdominal systems. Unlike CT-scanning (Computed Tomography), MRI can take scans from any angle. Figure 1 shows the well-known closed cylindrical MRI-scanner. For an extensive overview of MRI, see for example [1], [2].

The main magnet of an MRI-scanner generates a strong static magnetic field. Hydrogen nuclei in the body start spinning and align in this field. These nuclei respond to bursts of additional magnetic fields or RF-pulses (i.e. radio frequency pulses) with an MR-signal. Different types of tissues produce different signals. This forms the basis of MRI contrast. For an image of a cross-section of a body, a slice has to be selected within the body. For that, gradient coils are installed. In the gradient coils, dynamic currents are generated, inducing a dynamic magnetic field. A gradient coil is intended to create a gradient in the magnetic field. Three different types of gradient coils, the so-called $x$-, $y$ - and $z$-coil, vary the magnetic field strength around the center in the corresponding directions. The gradients provoke a body 


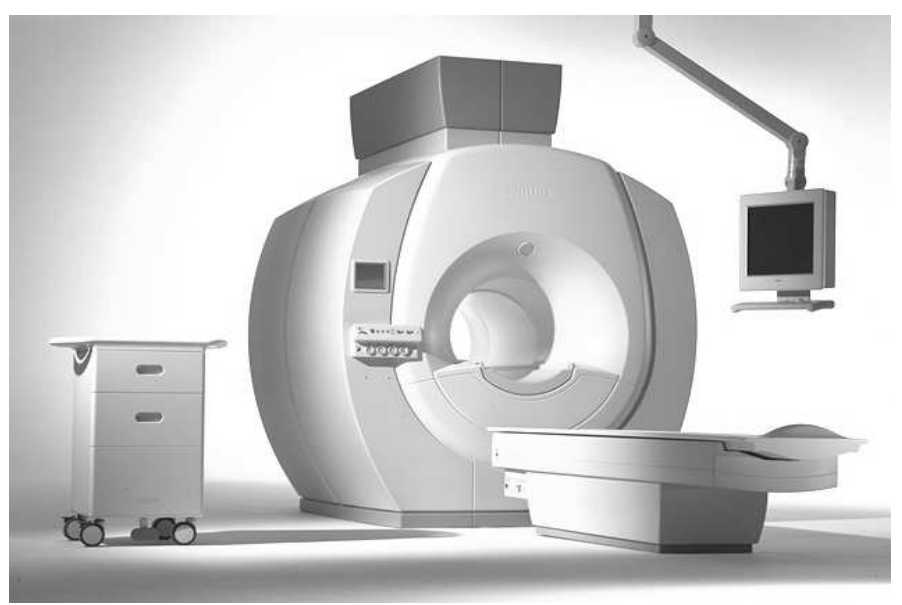

Figure 1: The closed cylindrical MRI-scanner

with spinning nuclei at varying frequencies. Only from the slice with a certain spin frequency, resonance signals are detected. The coils can be used for slices in different directions. To determine the position within a slice, the other gradients are used. For the design of gradient coils, the self-inductance plays an important role. A coil should have a self-inductance as small as possible, such that that it can be switched on and off quickly. A large inductance reduces the switching speed substantially.

One of the major problems in the use of switched gradient coils is the interaction of the rapidly changing fields with the conducting structures in the MRI-scanner including the gradient coils themselves. A dynamic magnetic field produced by a gradient coil induces eddy currents in the conducting structures. The eddy currents cause perturbations on the expected gradient field, leading to blurring in the MR picture, so they should be avoided as much as possible. This makes the role of eddy currents very important in the design of gradient coils.

This paper is concerned with the mathematical modelling and numerical simulation of the current distribution in a gradient coil of given configuration, in particular the z-coil. The geometry of the model is given as a finite number of rings, which are considered very thin. The rings have one common central axis and are parallel. They have equal radii, but can have different widths, and they may be positioned at different mutual distances. The model consists of a set of distinct rings. However, the current in a subset of rings can be driven by one and the same source. The total current through each subset is prescribed. The distribution of the current in one ring is time and space dependent. We consider a time-harmonic source with a relatively small frequency (in the order of $\mathrm{kHz}$ ).

Our overall aim is the calculation of the electric current distribution in each ring and from that the resistance and self-inductance of the whole system, each of them depending on the applied frequency. In the mathematical analysis an integral equation is derived for the current distribution and the Galerkin method is used to solve this equation. The most important issue is the choice of the basis functions. Numerical implementation is needed to determine the coefficients for the basis functions. It turns out that Legendre polynomials as basis functions are the most appropriate choice: They provide a simple analytical expression for the inner products in the Galerkin method, such that little computation time is needed 
for numerical integration. This expression indicates a fast convergence; only a few Legendre polynomials are needed for an accurate description of the current distribution.

From the numerous books on electromagnetic fields, three books have to be mentioned that deal with eddy currents. The book by Tegopoulos and Kriezis [3] is concerned with analytical methods applied mainly to two-dimensional configurations. The book by Stoll [4] is concerned with both analytical and numerical methods, giving a wide spectrum of eddy current considerations; especially the finite difference method has been widely documented. The book by Lammeraner and Stafl [5] is devoted to the analytical approach only and covers one-dimensional configurations.

Research on eddy currents is mostly devoted to localizing the areas where they occur. From the devices in which eddy currents are desirable and therefore exploited, we mention the eddy current waste separation belt. Rem describes in [6] how eddy current separation is used in the recycling of non-ferrous metals from waste. Due to a changing magnetic field around a moving belt, eddy currents are induced in the metal particles, which cause these particles to repel from the belt. Other areas in which eddy currents are desirable are brake systems [7] and induction heaters [8]. In all these examples, the electromagnetic energy from the eddy currents is converted to work done by forces or heat dissipation.

In most electromagnetic machines, eddy currents are undesirable. An example of undesired presence of eddy currents is in Cathode-Ray Tube (CRT) deflection coils; see [9]. The CRT is the predominant display device for televisions and computers. Eddy currents in the deflection coils contribute to heat dissipation and ringing (annoying patterns on the screen). Another unfavorable aspect of eddy currents is generation of destructive forces [10]. In MRI applications, eddy currents do not only occur in the scanner itself, but they can also be induced in the patient. The patient might be heated locally due to energy dissipation in tissue. This phenomenon is measured by the Specific Absorption Rate (SAR); see e.g. [11], [12]. Also, nerves might be stimulated by the induced currents. This phenomenon is referred to as Peripheral Nerve Stimulation (PNS); see e.g. [13], [14].

The design of gradient coils is an important subject in the development of MRI-scanners. For the design of a z-coil, the principle of a Maxwell pair is used. The Maxwell pair consists of two circular loops of wire carrying a current in opposite direction; see also Section 4.2 in this paper. A straightforward approach to obtain a better gradient field is to introduce more loops or a coil with multiple turns. In [15], [16], methods are described to determine the positions of the loops and the intensities of the currents. Because of the anti-symmetry of the configuration, the field and all its even derivatives vanish at the origin. By properly selecting the positions and the currents, we can systematically remove the third, fifth and higher order odd derivatives, thus achieving a better gradient field.

In other methods, the values of the currents are fixed and the corresponding positions are computed. This leads to optimization methods, such as the conjugate gradient method [17] and the simulated annealing method [18]. Some basic methods for the design of $\mathrm{x}-$ and y-coils are described by [19], [20]. Another approach is the target field method [21]. In this approach the desired field is specified and the corresponding current distribution on the cylindrical surface is computed. Approaches using stream functions to determine optimal surface structures are reported among others by Peeren [22], [23], and Tomasi [24]. In [22], [23], the optimal structure is related to a minimization of the magnetic energy. In this approach, currents are assumed static. Possible displacements of the currents due to inductive effects are not taken into account. In [24], the current distribution is discretized by use of onedimensional wires. 
As a result of eddy currents, the gradient homogeneity can be degraded and the rise and decay times of the switched field can be increased. A solution to this problem is to place a shield between gradient coils and their surrounding structures, so that the gradient field is reduced to zero outside the shield. This technique is often referred to as passive shielding [25]. An even better solution is to design gradient coils that produce a low intensity magnetic field outside the coils and thus hardly induce eddy currents in other conducting structures. Such coils must consist of at least two coils of different sizes. The outer coil, i.e. the shield coil, produces a field that cancels the one of the primary coil outside the shield coil. This technique is referred to as active shielding [26], [27]. Another issue in gradient coil design is the reduction of disturbing sound. Some recent publications on acoustic control in gradient coils are e.g. by Mansfield et al. [28] and Li et al. [29].

In previous research, Ulicevic [30] modeled the edge effect in a gradient coil by use of infinitely long plane rectangular strips. This model was extended in [31], and forms the basis of the present work. The eddy currents in a set of rings have been determined by the author [32], [33] and the characteristics of the magnetic field induced by the resulting currents are investigated by Tas [34].

The analytical approach in this paper leads to an integral equation of the second kind for the current distribution. Atkinson [35], [36] proposed methods, such as the Nyström method and the collocation method to solve such an integral equation. The asymptotic behavior of the integral kernel is logarithmic and therefore, inspired by the paper of Reade [37], we use the Galerkin method with Legendre polynomials as the basis functions.

In Section 2, we derive the integral equation for the current distribution by applying the Maxwell equations to the geometry of thin conducting rings on a cylinder. The equations are written in dimensionless form and the behavior of the kernel function is investigated. In Section 3, we present the approach of the Galerkin method for the actual problem of the rings and the choice of the basis functions. In Section 4, the results of the numerical simulations are shown for different configurations. In Section 5, we summarize the conclusions that can be drawn from the analytical and numerical models. Furthermore, we give some recommendations for further research on eddy currents in gradient coils.

\section{Mathematical analysis}

\subsection{Model definition}

The z-coil consists of a long thin strip of copper wound around a cylinder. It has the shape of a set of parallel rings connected by short joints from one ring to the other. The electromagnetic effects of the joints in the copper are negligible due to another strip just below the joints, which carries a current that roughly cancels out possible disturbances. The width of the strip is a few centimeters and its thickness a few millimeters. The distance between two successive loops varies from a few millimeters to a few centimeters. For the model, we consider a finite number, $N$, of circular loops of strips, which are parallel and have the same central axis; see Figure 2. We use cylindrical coordinates $(r, \varphi, z)$, where the $z$-axis coincides with the central axis of the cylinder. The radius of the cylinder is $R$, and the surface of the cylinder, denoted as $S_{c}$, is defined by

$$
S_{c}=\left\{(r, \varphi, z) \in \mathbb{R}^{3} \mid r=R,-\pi \leq \varphi \leq \pi,-\infty<z<\infty\right\} .
$$




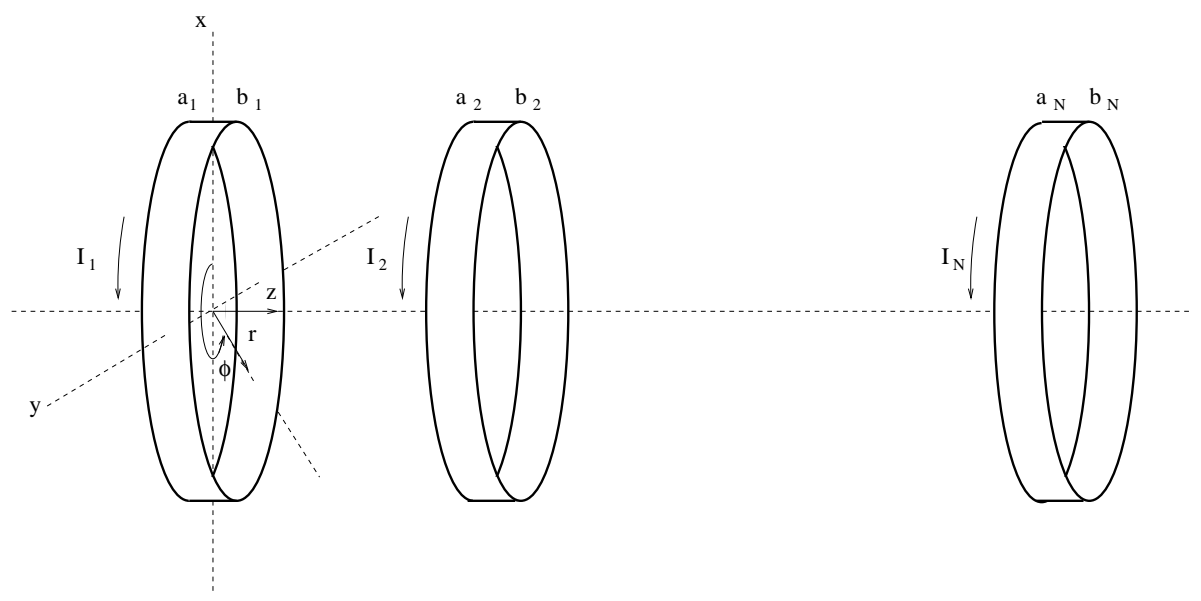

Figure 2: The geometry of the model, consisting of circular loops of strips.

Each separate strip is of uniform width. In our model, not all the strips have to be of the same width and the mutual distances may be different. The thickness $h$ is assumed to be the same for all strips.

Through the strips, a time-harmonic current of frequency $\omega$ is flowing, with current distribution

$$
\mathbf{J}(r, \varphi, z, t)=\operatorname{Re}\left\{\mathbf{J}(r, \varphi, z) e^{-i \omega t}\right\} .
$$

A typical measure for the thickness is $h=2.5 \cdot 10^{-3} \mathrm{~m}$. The cross-section of a strip is a narrow rectangle, meaning that the thickness $h$ is small compared to the width, but more relevant for us is the ratio of $h$ with respect to the penetration depth $\delta$. The penetration depth is characteristic for the distance in thickness direction over which the current density changes essentially. If $\delta$ is larger than half the width $h / 2$ of the ring, the current density is approximately uniform in thickness direction. In the latter case, we can assume that the strips are infinitely thin, and replace the current density $\mathbf{J}$ (in $\mathrm{A} / \mathrm{m}^{2}$ ) by the current per unit of length $\mathbf{j}$ (in $\mathrm{A} / \mathrm{m}$ ), defined as

$$
\mathbf{j}=\int_{R}^{R+h} \mathbf{J} \mathrm{d} r \approx h \mathbf{J}
$$

The penetration depth is defined as $\delta=\sqrt{2 / \mu \sigma \omega}$; see e.g. [38, Eq. (35.7)]. The values for the electric conductivity $\sigma$, the magnetic permeability $\mu=\mu_{0}$ and the electric permittivity $\epsilon=\epsilon_{0}$ are shown in Table 1. For the frequencies $f<10^{4} \mathrm{~Hz}$, we find that $\delta<h / 2$, such that the replacement of $\mathbf{J}$ by $\mathbf{j}=h \mathbf{J}$ is justified. We say then that the strip is infinitely thin. From now on, we refer to these infinitely thin circular loops of strips as rings.

The current distribution in the rings then is independent of $r(\mathbf{j}=\mathbf{j}(\varphi, z))$, and has no component in the $r$-direction $\left(j_{r}=0\right)$.

The rings occupy the surface $S \cup$ in space, described in cylindrical coordinates by

$$
S_{\cup}=\sum_{p=1}^{N} S_{p}, \quad S_{p}=\left\{(r, \varphi, z) \in \mathbb{R}^{3} \mid r=R,-\pi \leq \varphi \leq \pi, a_{p} \leq z \leq b_{p}\right\} .
$$


Table 1: Values of geometrical and material constants for copper rings.

\begin{tabular}{clll}
\hline Symbol & Name & Value & \\
\hline$\sigma$ & Electric conductivity & $5.8 \cdot 10^{7}$ & $\Omega^{-1} \mathrm{~m}^{-1}$ \\
\hline$\mu$ & Magnetic permeability & $4 \pi \cdot 10^{-7}$ & $\mathrm{Hm}^{-1}$ \\
\hline$\epsilon$ & Electric permittivity & $8.85 \cdot 10^{-12}$ & $\mathrm{Cm}^{-1}$ \\
\hline$h$ & Thickness of the rings & $2.5 \cdot 10^{-3}$ & $\mathrm{~m}$ \\
\hline
\end{tabular}

The total current through a ring is $I_{p}(t)=\operatorname{Re}\left\{I_{p} e^{-i \omega t}\right\}$, for $p=1, \ldots, N$, where each $I_{p} \in \mathbb{C}$ is a complex constant. By $G^{-}$, we define the inner region of the cylinder, and by $G^{+}$the outer region.

To obtain the mathematical description of our problem, we start from the Maxwell equations; see e.g. Stratton [39, Ch. 1]. All the media are non-polarizable and non-magnetizable. The copper rings are isotropic, homogeneous, linear conductors, in which Ohm's law holds. We consider only time-harmonic electromagnetic fields, i.e fields that uniformly contain the term $e^{-i \omega t}$; see (2). As all fields have this behavior, this term can be omitted in the equations, which then contain only the spatial fields. An electro quasi-static approach is applied, implying that the displacement current (i.e. the term $\partial \mathbf{D} / \partial t$ in Ampère's law) and the instationary term $\partial \rho / \partial t$ in the law of conservation of charge are neglected. The result of the latter neglect is a divergence-free current in the rings, i.e. $\nabla \cdot \mathbf{j}=0$. We denote by $\mathbf{H}$ the magnetic field strength, and by $\mathbf{E}$ the electric field strength. This leaves us with the Maxwell equations, valid in both $G^{-}$and $G^{+}$, (here, $\mathbf{E}=\mathbf{E}(\mathbf{x})=\mathbf{E}(r, \varphi, z)$, etc.)

$$
\nabla \times \mathbf{E}=i \omega \mu \mathbf{H}, \quad \nabla \cdot \mathbf{E}=\nabla \times \mathbf{H}=\nabla \cdot \mathbf{H}=0
$$

and, moreover, in the sheets

$$
\nabla \cdot \mathbf{j}=0, \quad \mathbf{j}=\mathbf{j}^{s}+\sigma h \mathbf{E},
$$

where $\mathbf{j}^{s}$ is the source current (see also (11), further on). The second equation of (6) is Ohm's law, generalized for including source currents; see e.g. [40, Eq. (2.1.4)], [41, Sect. 2.1]. At infinity we have the condition $\mathbf{H} \rightarrow \mathbf{0}$.

Being a surface current, the current $\mathbf{j}$ does not have a component in the $r$-direction, so the first equation of (6) becomes

$$
\frac{1}{R} \frac{\partial j_{\varphi}}{\partial \varphi}+\frac{\partial j_{z}}{\partial z}=0
$$

Due to the axial symmetry of the problem, all fields are $\varphi$-independent. In that case, (7) together with the boundary condition, saying that $j_{z}$ is zero at the edges of the rings, yields $j_{z} \equiv 0$. So, we are left with

$$
\mathbf{j}=j_{\varphi}(z) \mathbf{e}_{\varphi}
$$

Thus, we obtain two uncoupled sets of equations: one set for the components $\left\{E_{\varphi}, H_{r}, H_{z}\right\}$ and one set for the components $\left\{E_{r}, E_{z}, H_{\varphi}\right\}$. Only the first set is coupled with the current density via the boundary conditions. The second set is identically equal to zero.

The equations (5) - (6) are valid for ordinary points, i.e. for points in whose neighborhood the physical properties of the medium vary continuously. The points on the cylinder $S_{c}$ that are between the rings, are ordinary points. Points on the rings, i.e. on $S_{\cup}$, are not ordinary. 
As the thickness of the rings is negligibly small, the regions $G^{-}$and $G^{+}$are both bounded by $r=R$. From now on, we denote the field components in $G^{-}$as $\left\{E_{\varphi}^{-}, H_{r}^{-}, H_{z}^{-}\right\}$and in $G^{+}$as $\left\{E_{\varphi}^{+}, H_{r}^{+}, H_{z}^{+}\right\}$.

At $r=R$, the field components of $\mathbf{H}$ and $\mathbf{E}$ have jumps across the surface $S_{\cup}$. Denoting the jump across $S_{\cup}$ by $\llbracket \rrbracket$, we have

$$
\llbracket \mathbf{E} \times \mathbf{n} \rrbracket=\llbracket \mathbf{H} \cdot \mathbf{n} \rrbracket=0, \llbracket \mathbf{E} \cdot \mathbf{n} \rrbracket=Q_{s}, \quad \llbracket \mathbf{H} \times \mathbf{n} \rrbracket=-\mathbf{j},
$$

where $\mathbf{n}=\mathbf{e}_{r}$, and $Q_{s}$ is the surface charge. For our purposes $Q_{s}$ is irrelevant. Applying these boundary conditions to our model yields

$$
\begin{gathered}
H_{r}^{-}(R, z)=H_{r}^{+}(R, z), \quad E_{\varphi}^{-}(R, z)=E_{\varphi}^{+}(R, z), \\
H_{z}^{-}(R, z)-H_{z}^{+}(R, z)=j_{\varphi}(z)
\end{gathered}
$$

for each ring.

The set of equations is not complete yet. To create a current, we need a source. In our model, a source current $\mathbf{j}^{s}$ is applied. This current has a harmonic time behavior and its amplitude at the rings is prescribed. Due to this current, the electromagnetic fields change simultaneously and an extra current is induced. These induced currents are called eddy currents $\mathbf{j}^{e}$. The total spatial current $\mathbf{j}=j_{\varphi}(z) \mathbf{e}_{\varphi}$ is given by

$$
j_{\varphi}(z)=j_{\varphi}^{s}(z)+j_{\varphi}^{e}(z)
$$

Introducing a vector potential $\mathbf{A}$, defined by $\mathbf{B}=\nabla \times \mathbf{A}$, and a scalar potential $\Phi$, we can write

$$
\mathbf{E}=i \omega \mathbf{A}-\nabla \Phi .
$$

The scalar potential $\Phi$ must satisfy the Laplace equation (which follows from Gauss' law and the Coulomb gauge, $\nabla \cdot \mathbf{A}=0$ ) and must vanish at infinity. Therefore, it is identically equal to zero. The problem description in terms of the electric field $\mathbf{E}$ is transformed into a description in terms of the vector potential $\mathbf{A}$. Since only the $\varphi$-component of $\mathbf{E}$ is unequal to zero, also only the $\varphi$-component $A_{\varphi}=\left(\mathbf{A}, \mathbf{e}_{\varphi}\right)$ of $\mathbf{A}$ is non-zero here. Using Ohm's law (6) to eliminate $\mathbf{E}$ from (12), we obtain for $A_{\varphi}$ on a strip

$$
i \omega h \sigma A_{\varphi}(R, z)=j_{\varphi}(z)-j_{\varphi}^{s}(z), \quad z \in S_{\cup}
$$

where $j_{\varphi}$ is the total current on the strip.

We define a uniform source current on each of the rings. We assume that the rings are coupled in groups, and that each group is connected to one source. This means that for each group the function $j_{\varphi}^{s}(z)$ is uniform, i.e. $j_{\varphi}^{s}(z)=C_{l}$. So, for $L$ different groups, we have the constants $C_{l}, l=1, \ldots, L$. We express $j_{\varphi}^{s}(z)$ in characteristic functions $\psi_{l}(z)$, which are zero everywhere except on the rings of group $l$, where they have value one. Note that for the inner product of two characteristic functions $\psi_{l_{1}}$ and $\psi_{l_{2}}$, where $l_{1}, l_{2} \in\{1, \ldots, L\}$, one has

$$
\left(\psi_{l_{1}}(z), \psi_{l_{2}}(z)\right)=\int_{-\infty}^{\infty} \psi_{l_{1}}(z) \psi_{l_{2}}(z) \mathrm{d} z=D_{l_{1}} \delta_{l_{1} l_{2}}
$$

where $\delta_{l_{1} l_{2}}$ is the Kronecker delta function, which is equal to zero if $l_{1} \neq l_{2}$ and equal to one if $l_{1}=l_{2}$, and $D_{l_{1}}$ is the sum of the widths of all rings of group $l_{1}$. The function $j_{\varphi}^{s}(z)$ can now be written in the form

$$
j_{\varphi}^{s}(z)=\sum_{l=1}^{L} C_{l} \psi_{l}(z), \quad z \in S_{\cup} .
$$


To obtain a well-defined problem description, we still need $L$ extra relations, in order to determine the $L$ different constants $C_{1}, \ldots, C_{L}$. We require that the total current in each group of rings is prescribed, according to

$$
\int_{-\infty}^{\infty} j_{\varphi}(z) \psi_{l}(z) \mathrm{d} z=I_{l}, \quad l=1, \ldots, L
$$

\subsection{Dimensionless formulation}

For the dimension analysis, we introduce a characteristic current $j^{c}$ and a characteristic distance $D$, defined by

$$
D=R, \quad j^{c}=\frac{\sum_{l=1}^{L} I_{l}}{\sum_{l=1}^{L} D_{l}} .
$$

Thus, the distances are scaled by the radius of the cylinder, and the current is scaled by the average current through all rings. We obtain the following set of equations, for $A_{\varphi}, H_{r}, H_{z}$ and $j_{\varphi}$ (dimensionalized in an obvious way),

$$
\begin{array}{cc}
\frac{\partial A_{\varphi}}{\partial z}=-H_{r}, & \frac{1}{r} \frac{\partial}{\partial r}\left(r A_{\varphi}\right)=H_{z} \\
\frac{\partial H_{r}}{\partial z}-\frac{\partial H_{z}}{\partial r}=0, & \frac{1}{r} \frac{\partial}{\partial r}\left(r H_{r}\right)+\frac{\partial H_{z}}{\partial z}=0,
\end{array}
$$

with the boundary conditions

$$
\begin{gathered}
H_{r}^{-}(1, z)=H_{r}^{+}(1, z), \quad A_{\varphi}^{-}(1, z)=A_{\varphi}^{+}(1, z), \\
H_{z}^{-}(1, z)-H_{z}^{+}(1, z)=j_{\varphi}(z),
\end{gathered}
$$

and, from (13) and (15)

$$
i \kappa A_{\varphi}^{-}(1, z)=i \kappa A_{\varphi}^{+}(1, z)=j_{\varphi}(z)-\sum_{l=1}^{L} C_{l} \psi_{l}(z)
$$

where

$$
\kappa=h \sigma \mu \omega R \text {. }
$$

Finally, the expression for the total current (16) becomes in dimensionless form

$$
\int_{S \cup} j_{\varphi}(z) \psi_{l}(z) \mathrm{d} z=\frac{I_{l}}{j^{c} R}=\hat{I}_{l},
$$

for all $l=1, \ldots, L$. Note that the functions $\psi_{l}(z)$ now represent the characteristic functions of group $l$, referring to the dimensionless widths of the strips. This means that (14) turns into

$$
\left(\psi_{l_{1}}, \psi_{l_{2}}\right)=\frac{D_{l_{1}}}{R} \delta_{l_{1} l_{2}}
$$

At infinity, the condition $\mathbf{H} \rightarrow \mathbf{0}$ remains unchanged. 


\subsection{Derivation of the integral formula for the current distribution}

Combining the equations of (18), we obtain for the vector potential

$$
\frac{\partial^{2} A_{\varphi}}{\partial z^{2}}+\frac{1}{r} \frac{\partial}{\partial r}\left(r \frac{\partial A_{\varphi}}{\partial r}\right)-\frac{1}{r^{2}} A_{\varphi}=0 .
$$

This equation is valid for both the inner region $G^{-}$and the outer region $G^{+}$. We solve (24) by applying Fourier transformation with respect to the variable $z$. Introducing the Fourier transforms $\hat{A}_{\varphi}^{ \pm}(r, p)=\mathcal{F}_{z}\left\{A_{\varphi}^{ \pm}(r, \cdot)\right\}(p)$, we transform (24) into

$$
-p^{2} \hat{A}_{\varphi}^{ \pm}+\frac{1}{r} \frac{\partial}{\partial r}\left(r \frac{\partial \hat{A}_{\varphi}^{ \pm}}{\partial r}\right)-\frac{1}{r^{2}} \hat{A}_{\varphi}^{ \pm}=0 .
$$

The general solution of $(25)$ is

$$
\hat{A}_{\varphi}^{-}(r, p)=a(p) I_{1}(|p| r), \quad \hat{A}_{\varphi}^{+}(r, p)=b(p) K_{1}(|p| r),
$$

where, $I_{1}$ and $K_{1}$ are the modified Bessel functions of order one of the first and second kind, respectively. The coefficients $a(p)$ and $b(p)$ can be found from the boundary conditions (19), transformed to the Fourier domain. For this, we first determine $H_{r}$ and $H_{z}$ from (18). Using $\hat{\jmath}_{\varphi}(p)=\mathcal{F}_{z}\left\{j_{\varphi}(\cdot)\right\}(p)$ and the Wronskian $W\left(K_{0}, I_{0}\right)=I_{0}(|p|) K_{1}(|p|)+I_{1}(|p|) K_{0}(|p|)=1 /|p|$, see [42, Eq. (9.6.15)], we obtain

$$
\hat{A}_{\varphi}^{-}(r, p)=I_{1}(|p| r) K_{1}(|p|) \hat{\mathrm{j}}_{\varphi}(p), \quad \hat{A}_{\varphi}^{+}(r, p)=K_{1}(|p| r) I_{1}(|p|) \hat{\mathrm{j}}_{\varphi}(p) .
$$

For the inverse Fourier transform, we use the convolution principle to obtain

$$
A_{\varphi}^{-}(r, z)=\int_{-\infty}^{\infty} j_{\varphi}(\zeta) \mathcal{K}_{1}(r, z-\zeta) \mathrm{d} \zeta, \quad A_{\varphi}^{+}(r, z)=\int_{-\infty}^{\infty} j_{\varphi}(\zeta) \mathcal{K}_{2}(r, z-\zeta) \mathrm{d} \zeta
$$

where

$$
\mathcal{K}_{1}(r, z)=\frac{1}{\pi} \int_{0}^{\infty} I_{1}(p r) K_{1}(p) \cos p z \mathrm{~d} p, \quad r<1
$$

and

$$
\mathcal{K}_{2}(r, z)=\frac{1}{\pi} \int_{0}^{\infty} K_{1}(p r) I_{1}(p) \cos p z \mathrm{~d} p, \quad r>1 .
$$

Only on the rings the current is unequal to zero, such that the integrals in (28) have contributions over the surface $S_{\cup}$ only.

Finally, we substitute the solution for the vector potential on $r=1$ into (20) to arrive at the integral equation for $j_{\varphi}(z)$ we are looking for:

$$
j_{\varphi}(z)-i \kappa \int_{S \cup} j_{\varphi}(\zeta) \mathcal{K}(z-\zeta) \mathrm{d} \zeta=\sum_{l=1}^{L} C_{l} \psi_{l}(z), \quad z \in S_{\cup}
$$

where

$$
\mathcal{K}(z)=\lim _{r \uparrow 1} \mathcal{K}_{1}(r, z)=\lim _{r \downarrow 1} \mathcal{K}_{2}(r, z)=\frac{1}{\pi} \int_{0}^{\infty} I_{1}(p) K_{1}(p) \cos p z \mathrm{~d} p .
$$




\subsection{Characteristics of the kernel function}

For further computations we need to investigate the behavior of the kernel function $\mathcal{K}(z)$. For this, we can use either of the following expressions:

$$
\begin{aligned}
\mathcal{K}(z) & =\frac{1}{\pi} \int_{0}^{\infty} I_{1}(p) K_{1}(p) \cos p z \mathrm{~d} p=\frac{1}{2} \int_{0}^{\infty} J_{1}^{2}(p) \mathrm{e}^{-p z} \mathrm{~d} p \\
& \left.=\frac{1}{4|z|^{3}}{ }_{2} F_{1}\left(\frac{3}{2}, \frac{3}{2} ; 3 ;-\frac{4}{z^{2}}\right)=\frac{1}{2 \pi k}\left[\left(2-k^{2}\right) K(k)-2 E(k)\right)\right] .
\end{aligned}
$$

Here, $J_{1}$ is the Bessel function of order one of the first kind (see [43]), ${ }_{2} F_{1}$ is the hypergeometric function (see [44]), $K$ and $E$ are the complete elliptic integrals of the first and second kind, respectively (see [45]), and $k^{2}=4 /\left(4+z^{2}\right)$.

The reason for presenting these four expressions is that either one of them is used in literature and that different authors have different preferences. Apart from the ones in (33), other expressions can be used as well. However, the expressions in (33) are the more common in the symbolic toolboxes of programs, such as Mathematica and Maple.

We note that the last expression of (33), i.e. the one with the elliptic functions, is similar to the formula for the mutual inductance between two circular loops of wires; see e.g. [46], [32], or [47]. It is shown by the first author in [32], that the model of a set of circular loops of wires provides results for discrete currents, which describe the qualitative behavior of the continuous current distribution in rings with a similar geometry. The wire model yields some insight in the problem, but cannot be used as a proper quantitative validation, because the thickness and the number of wires are arbitrary.

Investigation of the behavior of the kernel function $\mathcal{K}(z)$ reveals that it has a singularity in $z=0$. From a numerical point of view, it is important to find an approximate formula that is valid for small $z$. The asymptotic expansion of $\mathcal{K}(z)$ for $z \rightarrow 0$ up to terms of $\mathcal{O}\left(z^{2}\right)$ is of the form

$$
\mathcal{K}(z)=-\frac{1}{2 \pi}\left(\log |z|-\log 2+\Psi^{(0)}\left(\frac{3}{2}\right)+\gamma\right)+\mathcal{O}\left(z^{2}\right),
$$

where $\Psi^{(0)}(3 / 2) \approx 0.03649\left(\Psi^{(0)}\right.$ is the polygamma function; see [42, Eq. (6.4.4)]), and $\gamma \approx 0.57722$ (Euler's constant; see [42, Eq. (6.1.3)]). In Figure 3 both the kernel function (solid line) and the series expansion (dashed line) are drawn for $z \in[-4,4]$. The difference between $\mathcal{K}(z)$ and the series expansion is a regular function, which is exactly equal to zero in $z=0$ (both its value and its first derivative). In the numerical computations we make use of the approximation for $\mathcal{K}(z)$ according to (34).

That the kernel function can be split up into a logarithmic part and a regular part enables us to investigate the equivalence between a set of parallel rings and a set of infinitely long parallel strips in one plane. For the strips in one plane, the integral formulation is derived in [48] and it has a kernel that is purely logarithmic. Therefore, according to (34), the solution of the ring problem is partly accounted for by the solution of the plane-strip problem. The

remaining part of the solution is determined from the regular difference function, i.e. the difference between $\mathcal{K}(z)$ and the $\log |z|$ term. 


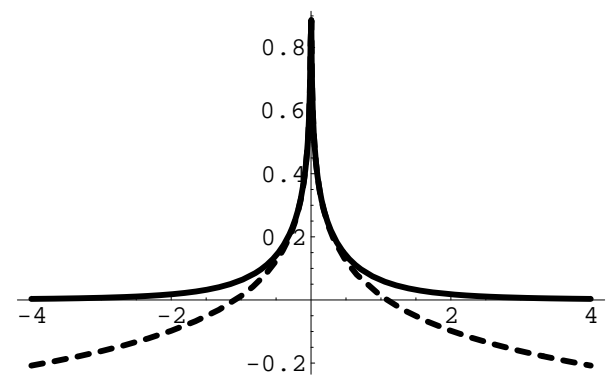

Figure 3: The kernel $\mathcal{K}(z)$ (solid line) and the approximate function (dashed line).

\section{Choice of the basis functions}

We introduce the operator $\mathrm{K}$ by

$$
(\mathrm{K} f)(z)=\int_{S \cup} f(\zeta) \mathcal{K}(z-\zeta) \mathrm{d} \zeta
$$

and we define the projection $\mathcal{P}$ on the linear span of the characteristic functions $\psi_{l}, l \in$ $\{1, \ldots, L\}$ by

$$
(\mathcal{P} f)(z)=\sum_{l=1}^{L} \frac{\left(f, \psi_{l}\right)}{\left(\psi_{l}, \psi_{l}\right)} \psi_{l}(z)
$$

The projection applied to $j_{\varphi}$ yields, with $(22)$ and (23)

$$
\mathcal{P} j_{\varphi}=\sum_{l=1}^{L} \frac{R}{D_{l}}\left(j_{\varphi}, \psi_{l}\right) \psi_{l}=\sum_{l=1}^{L} \frac{R \hat{I}_{l}}{D_{l}} \psi_{l} .
$$

Introducing $j_{\perp}=(\mathcal{I}-\mathcal{P}) j_{\varphi}$, to eliminate $j_{\varphi}$ from (31) in favor of $j_{\perp}$, and next applying the operator $(\mathcal{I}-\mathcal{P})$ to the thus obtained equation, we arrive at

$$
j_{\perp}-i \kappa(\mathcal{I}-\mathcal{P}) \mathrm{K}_{\perp}=i \kappa \sum_{l=1}^{L} \frac{R \hat{I}_{l}}{D_{l}}(\mathcal{I}-\mathcal{P}) \mathrm{K} \psi_{l} .
$$

Hence, the unknown constants $C_{l}$ have disappeared (this is due to $(\mathcal{I}-\mathcal{P}) \psi_{l}=0$ ), and are replaced by the prescribed currents $\hat{I}_{l}$.

We approximate $j_{\perp}$ by a finite series of basis functions $\phi_{m}(z)$, according to

$$
j_{\perp}(z) \doteq \sum_{m=1}^{M} \alpha_{m} \phi_{m}, \quad \phi_{m} \in \operatorname{ran}(\mathcal{I}-\mathcal{P}) .
$$

Substituting (39) into (38), taking the inner product of this relation with $\phi_{n}$, and using the self-adjointness of the operator $(\mathcal{I}-\mathcal{P})$, we obtain

$$
\sum_{m=1}^{M} \alpha_{m}\left(\phi_{m}, \phi_{n}\right)-i \kappa \sum_{m=1}^{M} \alpha_{m}\left(\mathrm{~K} \phi_{m}, \phi_{n}\right)=i \kappa \sum_{l=1}^{L} \frac{R \hat{I}_{l}}{D_{l}}\left(\mathrm{~K} \psi_{l}, \phi_{n}\right), \quad n=1,2, \ldots, M
$$


From this system of $M$ linear equations, the $M$ unknown coefficients $\alpha_{m}$ can be determined. For convenience of notation, we write (40) in a matrix formulation as

$$
(\mathbf{G}-i \kappa \mathbf{A}) \mathbf{a}=i \kappa \mathbf{B f},
$$

where $(m, n=1, \ldots, M ; l=1, \ldots, L)$

$$
\begin{aligned}
A(n, m) & =\left(\mathrm{K} \phi_{m}, \phi_{n}\right), \\
G(n, m) & =\left(\phi_{m}, \phi_{n}\right), \\
a(m) & =\alpha_{m}, \\
B(n, l) & =\left(\mathrm{K} \psi_{l}, \phi_{n}\right), \\
f(l) & =\frac{R \hat{I}_{l}}{D_{l}}
\end{aligned}
$$

The basis functions used to approximate the current distribution $j_{\perp}(z)$ are chosen to be Legendre functions. The reasons for this are:

- As shown in (34), the kernel of the integral has a logarithmic singularity. If we only consider the logarithmic part of the operator, then an analytical solution can be derived for the integral of the logarithmic function in combination with Legendre polynomials. The analytical solution is shown in (57). The regular part can be computed numerically straightforwardly.

- Due to the use of the analytical expression, the computations are fast.

- The analytical expression shows that the coefficients of the Legendre functions yield a fast convergence.

- We expect a current distribution with higher densities along the edges of a ring. Legendre functions also act particularly near the edges of their domain.

For the current distribution $j_{\perp}(z)$, the pertinent intervals are $\left[z_{0}^{(q)}, z_{1}^{(q)}\right], q=1, \ldots, N$, instead of $[-1,1]$. Therefore, we introduce shifted and scaled Legendre polynomials, $P_{s, q}\left(z ; c_{q} ; d_{q}\right)$, defined as

$$
P_{s, q}\left(z ; c_{q} ; d_{q}\right)=P_{s}\left(\frac{z-c_{q}}{d_{q}}\right), \quad z \in\left[a_{q}, b_{q}\right]
$$

where

$$
c_{q}=\frac{b_{q}+a_{q}}{2}, \quad d_{q}=\frac{b_{q}-a_{q}}{2}, \quad q=1, \ldots, N .
$$

Here, $a_{q}$ and $b_{q}$ are the axial begin and end positions of the rings (scaled on $R$ ), respectively. The shifted and scaled Legendre polynomials are used to construct the basis functions. We create a series expansion in different basis functions for each ring, separately. So,

$$
j_{\perp}=\sum_{s=1}^{S} \sum_{q=1}^{N} \alpha_{s, q} \phi_{s, q}+\sum_{q=1}^{N-L} \alpha_{0, q} \phi_{0, q},
$$

where $S$ denotes the number of degrees of Legendre functions that is included, and $N$ is the number of rings. The reason for splitting up the sum into two terms - one with $\phi_{s, q}$ and one with $\phi_{0, q}$ - follows below. We note that the total number of basis functions is $(S+1) N-L$, 
which will be set equal to the number $M$ used in (39). A requirement for the basis functions is that they are mutually orthogonal as well as orthogonal to the functions $\psi_{l}(z)$. For Legendre polynomials of degree one and higher, we have

$$
\int_{S \cup} P_{s, q}\left(z ; c_{q} ; d_{q}\right) \psi_{l}(z) \mathrm{d} z=0,
$$

for $q=1, \ldots, N, l=1, \ldots, L$, and $s \geq 1$. Therefore, we can choose the basis functions of degree one and higher equal to the shifted and scaled Legendre polynomials

$$
\phi_{s, q}=P_{s, q}\left(z ; c_{q} ; d_{q}\right), \quad q=1, \ldots, N, s=1, \ldots, S .
$$

The Legendre polynomial of degree zero is a constant function of value one. The total current $j_{\varphi}(z)$ is approximated for a part by $L$ constant functions $\psi_{l}(z)$. So, for $j_{\perp}(z), N-L$ constant functions are left, that can differentiate the current distributions in the rings within each group. Here, $\phi_{0, q}(z)$, denote the constant basis functions, which are mutually orthogonal and orthogonal to all other basis functions. This explains why we split off the sum with the $N-L$ terms in (49).

We can now express (42) - (46) as follows:

$$
\begin{aligned}
A(n, m) & =d_{q} d_{q^{\prime}} \int_{-1}^{1} \int_{-1}^{1} P_{s^{\prime}}(z) P_{s}(\zeta) \mathcal{K}\left(d_{q^{\prime}} z-d_{q} \zeta+c_{q^{\prime}}-c_{q}\right) \mathrm{d} \zeta \mathrm{d} z \\
G(n, m) & =\frac{2 d_{q}}{2 s+1} \delta_{m n} \\
a(m) & =\alpha_{m} \\
B(n, l) & =d_{q} d_{q^{\prime}} \int_{-1}^{1} \int_{-1}^{1} P_{s^{\prime}}(z) \mathcal{K}\left(d_{q^{\prime}} z-d_{q} \zeta+c_{q^{\prime}}-c_{q}\right) \mathrm{d} \zeta \mathrm{d} z \\
f(l) & =\frac{I_{l} \sum_{l=1}^{L} D_{l}}{D_{l} \sum_{l=1}^{L} I_{l}}
\end{aligned}
$$

with $m=(s-1) N+q$, and $n=\left(s^{\prime}-1\right) N+q^{\prime}$.

The elements of the matrices $\mathbf{A}$ and $\mathbf{B}$ can be determined from the integrals (51) and (54), whose integrands are only singular if $q=q^{\prime}$. We have already shown in Section 2.4 that this singularity is logarithmic. We split off the logarithmic part (34) and are left with a regular difference function. This difference function in combination with the Legendre polynomials can be integrated numerically straightforwardly. For the logarithmic part, together with the Legendre polynomials, an analytical expression exists; see [49]. Defining

$$
\Pi_{k k^{\prime}}=\frac{d_{q}^{2}}{2 \pi} \int_{-1}^{1} \int_{-1}^{1} P_{k}(z) P_{k^{\prime}}(z)\left[\log \left(d_{q}|z-\zeta|\right)-\log 2+\Psi^{(0)}\left(\frac{3}{2}\right)+\gamma\right] \mathrm{d} \zeta \mathrm{d} z,
$$

we obtain

$$
\Pi_{k k^{\prime}}= \begin{cases}\frac{8 d_{q}^{2}}{2 \pi\left(k+k^{\prime}\right)\left(k+k^{\prime}+2\right)\left[\left(k-k^{\prime}\right)^{2}-1\right]}, & \text { if } k+k^{\prime}>0 \text { even }, \\ 0, & \text { if } k+k^{\prime} \text { odd } \\ \frac{d_{q}^{2}}{2 \pi}\left(4 \log d_{q}-6+4 \Psi^{(0)}\left(\frac{3}{2}\right)+4 \gamma\right), & \text { if } k=k^{\prime}=0 .\end{cases}
$$

For different strips, i.e. $q \neq q^{\prime}$, no singularities occur. 


\section{Numerical Results}

\subsection{One ring}

We first consider one ring of width $D=4 \mathrm{~cm}$, positioned with its center at $z=0$. The thickness is $h=2.5 \mathrm{~mm}$, the radius is $R=35 \mathrm{~cm}$ and the amplitude of the total current through the ring is $I=600 \mathrm{~A}$. All values are typical values for a gradient coil. The current distribution is expanded in a series of Legendre polynomials, with coefficients calculated from the linear set of equations (41).

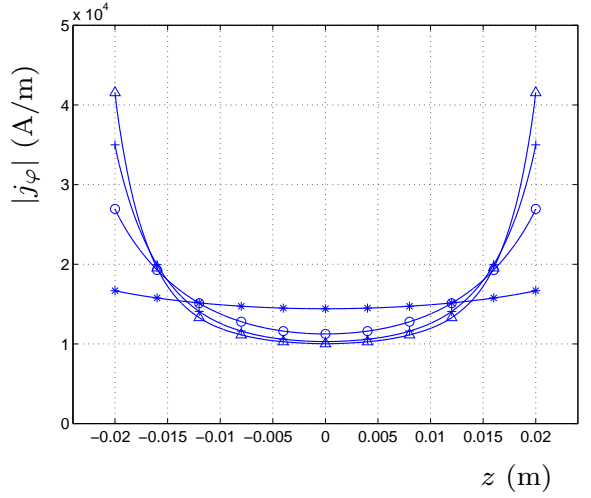

(a)

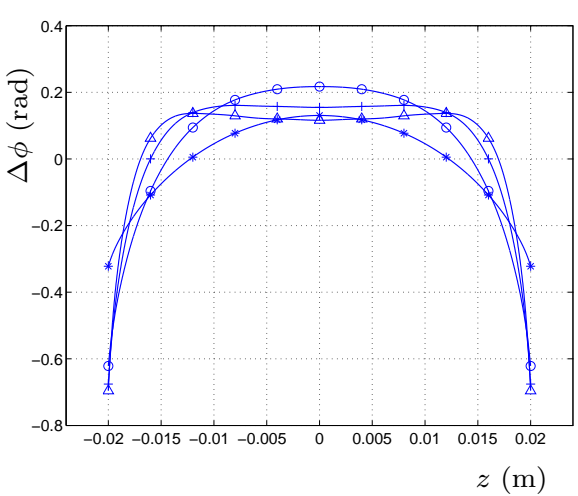

(b)

Figure 4: One ring of $4 \mathrm{~cm}$ width with a total current of $600 \mathrm{~A}$ at frequencies $f=100 \mathrm{~Hz}(*), f=400 \mathrm{~Hz}(\circ), f=700 \mathrm{~Hz}(+), f=1000 \mathrm{~Hz}$ $(\triangle)$. (a) Amplitude of the current distribution; (b) Phase-lag with respect to the source current.

Figure 4 (a) shows the amplitude of the current distribution in the ring for the four frequencies $f=100,400,700,1000 \mathrm{~Hz}$. We observe that the current at the edges is higher than in the center. This edge-effect, which is due to the self-inductance of the ring, becomes stronger as the frequency increases. For low frequencies, the current is distributed almost uniformly, whereas for high frequencies the current is more concentrated at the edges. The average current is always equal to $1.5 \cdot 10^{4} \mathrm{~A} / \mathrm{m}$, which is the value for $j^{c}=I / D$.

To obtain the time-dependent result for the currents, we multiply the current density by $\mathrm{e}^{-i \omega t}$ and take the real part. Simulating this case, we observe a phase difference in the system. In Figure 4 (b), the phase-lag $\Delta \phi$ with respect to the source current is shown as function of the position for the four frequencies $f=100,400,700,1000 \mathrm{~Hz}$. For each frequency only two points are in phase with the source (i.e. $\Delta \phi=0$ ). The currents near the edges are ahead in phase with respect to the source (i.e. negative phase-lag), whereas currents at the center are behind in phase. The phase difference also varies with the frequency.

We note that the system is linear with respect to the magnitude of the current, which means that if we increase the total current $I$, then the current densities are increased proportionally. The shapes of the distributions in Figures 4 (a) and (b) remain the same, but scales are changing. 


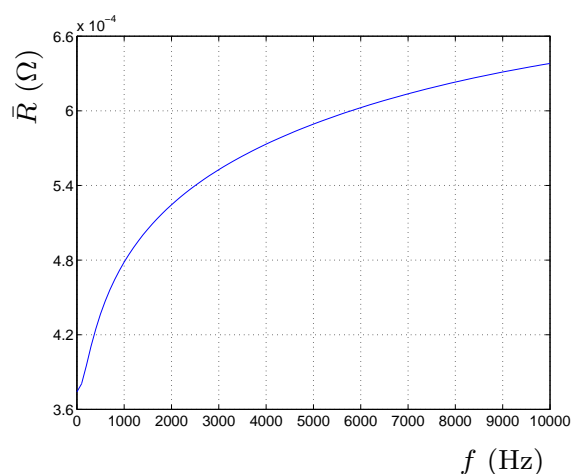

(a)

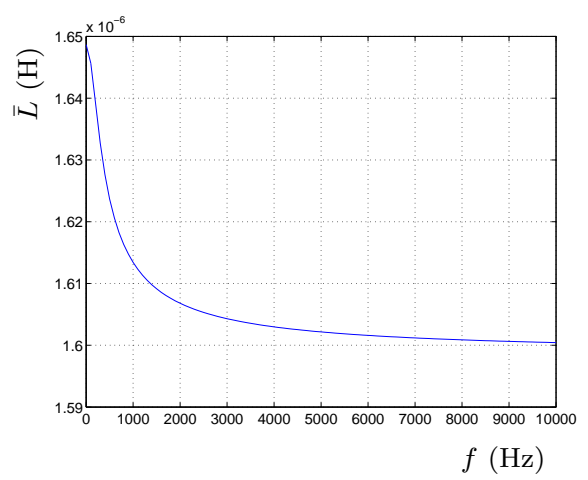

(c)

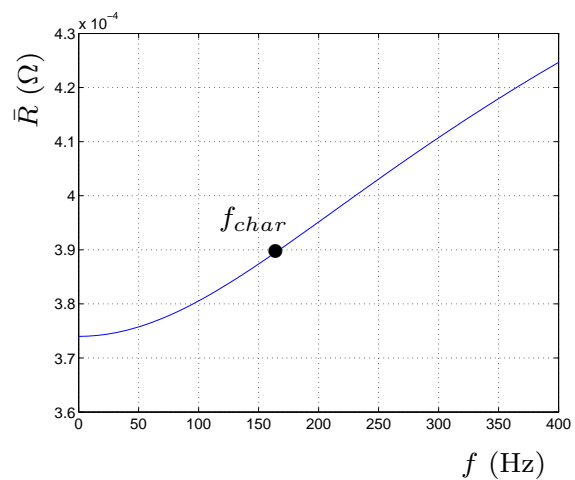

(b)

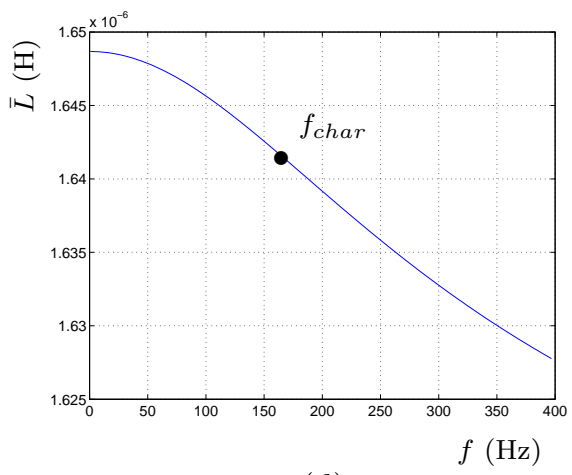

(d)

Figure 5: One ring of $4 \mathrm{~cm}$ width with a total current of $600 \mathrm{~A}$. (a) Resistance $\bar{R}$ at frequencies up to $f=10000 \mathrm{~Hz}$; (b) Resistance $\bar{R}$ at frequencies up to $f=400 \mathrm{~Hz}$; (c) Self-inductance $\bar{L}$ at frequencies up to $f=10000 \mathrm{~Hz}$; (d) Self-inductance $\bar{L}$ at frequencies up to $f=400 \mathrm{~Hz}$. 
The first characteristic quantity we present, is the resistance $\bar{R}$. It is defined as the time-averaged dissipated power, $\bar{P}_{\text {diss }}$, divided by the squared effective current, $I_{e}^{2}$,

$$
\bar{R}=\frac{\bar{P}_{\text {diss }}}{I_{e}^{2}}=\frac{\int_{\mathcal{V}} \mathbf{J} \cdot \mathbf{J}^{*} \mathrm{~d} v}{\sigma I I^{*}} .
$$

Here, $\mathcal{V}$ denotes the dimensional volume of the ring and an asterisk stands for the complex conjugate. We have computed $\bar{R}$ for different frequencies. In Figure 5 (a), $\bar{R}$ is shown for the frequency range $[0,10000] \mathrm{Hz}$. We observe that $\bar{R}$ increases with the frequency. The physical interpretation is that due to the stronger edge-effects the current flows through a narrower part of the ring, leading to a higher resistance. The resistance $\bar{R}_{D C}$ at $f=0 \mathrm{~Hz}$ is verified analytically by taking the circumferential length divided by the conductivity times the cross-sectional surface, i.e.

$$
\bar{R}_{D C}=\frac{2 \pi R}{h D \sigma} \approx 3.74 \cdot 10^{-4} \Omega .
$$

We observe a decay in the slope of the resistance. In Smythe [47, pp. 368-371], it is shown how a similar behavior occurs in the situation of a conducting medium filling a half-space, with a harmonic varying source current flowing along the surface. The induced current causes a resistance that increases with the square root of the frequency. This can be explained by the decrease of the order of the edge effect for increasing frequency. In the case of a thin ring, the decay of the slope is stronger. The graph in Figure 5 (a) can be fitted by a function that is proportional to the frequency to the power one eighth.

In Figure 5 (b), the resistance is shown for the smaller range of frequencies up to $f=400$ $\mathrm{Hz}$, with a higher resolution than used in the range from 0 to $10000 \mathrm{~Hz}$. We observe that the behavior of $\bar{R}$ changes from low to high frequency range. We observe a point of inflection $f_{\text {char }}$ close to $170 \mathrm{~Hz}$. Such a point of inflection is also observed in the next example, a ring of $30 \mathrm{~cm}$ width. We discuss this phenomenon at the end of this section.

The next characteristic quantity we consider, is the self-inductance $\bar{L}$. It is defined as the time-averaged magnetic energy, $\bar{U}_{m}$, divided by half the squared effective current,

$$
\bar{L}=\frac{\bar{U}_{m}}{\frac{1}{2} I_{e}^{2}}=\frac{\int_{\mathcal{V}} \mathbf{A} \cdot \mathbf{J}^{*} \mathrm{~d} v}{I I^{*}} .
$$

In Figure 5 (c), the self-inductance $\bar{L}$ is shown for frequencies up to $f=10000 \mathrm{~Hz}$. We see that $\bar{L}$ decreases with the frequency. Thus, if the current is jostled near the edges of the ring, as happens in the case of higher frequencies, then the inductive effects decrease. Or, conversely, if the current changes more rapidly in time, then the self-inductance has to be lower. For that, the current starts flowing more near the edges of the ring than at the center.

For low frequency range, from 0 to $400 \mathrm{~Hz}$, we observe that the self-inductance $\bar{L}$ changes behavior as frequencies increase; see Figure $5(\mathrm{~d})$. The inflection is at the same characteristic frequency $f_{\text {char }}$ as found for the resistance.

Apart from a dependence on the frequency, the results of our simulations also depend on the width $D$ of the ring. To illustrate this, we repeat the computations for a ring of $30 \mathrm{~cm}$ width. Figures 6 (a) and (b) show the amplitude of the current density and the phase-lag in this ring for the four frequencies $f=100,400,700,1000 \mathrm{~Hz}$. Here, the average current is equal to $2.0 \cdot 10^{3} \mathrm{~A} / \mathrm{m}$, i.e. the value for $j^{c}=I / D$. We observe that the current is more concentrated near the edges than in the $4 \mathrm{~cm}$ ring. 


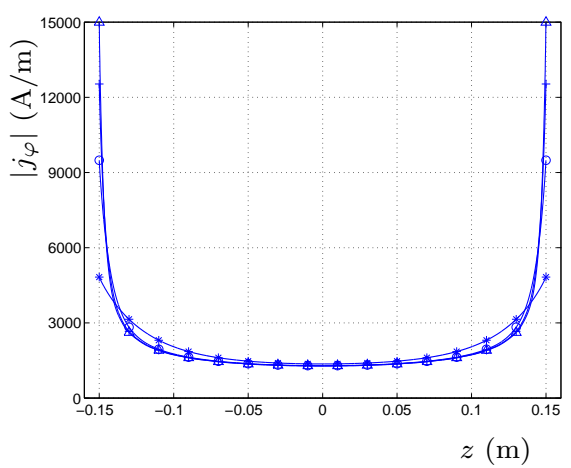

(a)

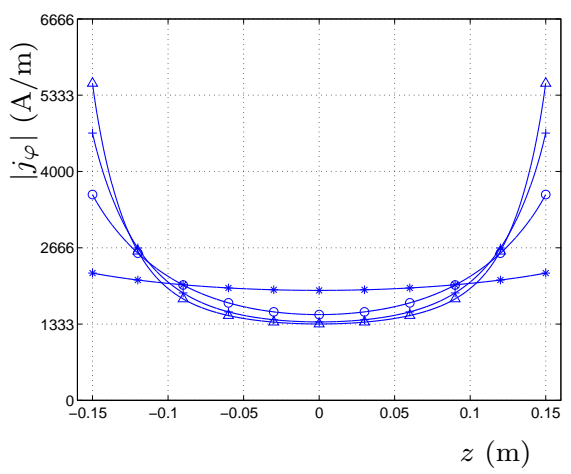

(c)

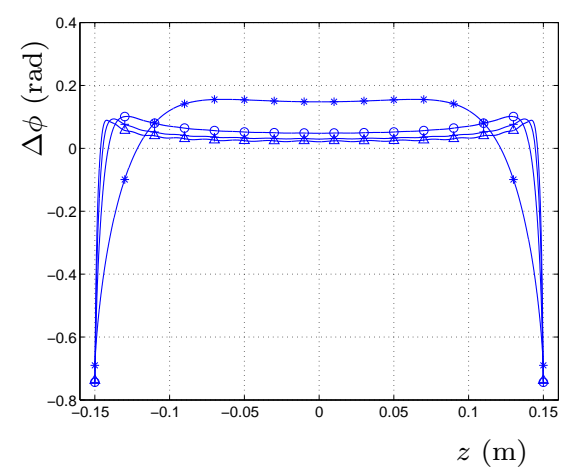

(b)

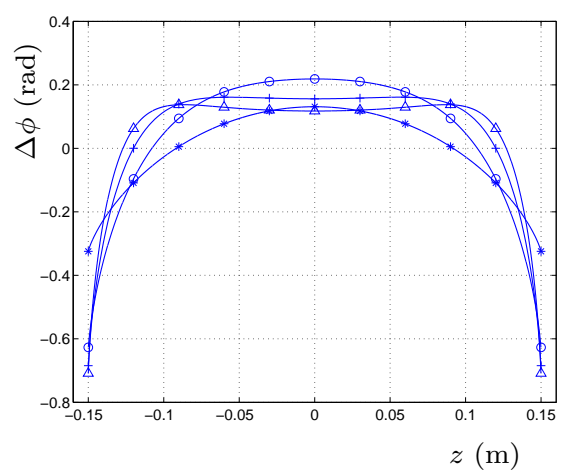

(d)

Figure 6: One ring of $30 \mathrm{~cm}$ width with a total current of $600 \mathrm{~A}$. (a) Amplitude of the current distribution at frequencies $f=100 \mathrm{~Hz}(*)$, $f=400 \mathrm{~Hz}($ o), $f=700 \mathrm{~Hz}(+), f=1000 \mathrm{~Hz}(\triangle)$; (b) Phase-lag with respect to the source current at frequencies $f=100 \mathrm{~Hz}(*)$, $f=400 \mathrm{~Hz}(\circ), f=700 \mathrm{~Hz}(+), f=1000 \mathrm{~Hz}(\triangle)$; (c) Amplitude of the current distribution at frequencies $f=13.3 \mathrm{~Hz}(*)$, $f=53.3 \mathrm{~Hz}(\circ), f=93.3 \mathrm{~Hz}(+), f=133.3 \mathrm{~Hz}(\Delta)$; (b) Phase-lag with respect to the source current at frequencies $f=13.3 \mathrm{~Hz}(*)$, $f=53.3 \mathrm{~Hz}(\circ), f=93.3 \mathrm{~Hz}(+), f=133.3 \mathrm{~Hz}(\triangle)$. 
Let us take a closer look at the integral equation

$$
(\mathrm{I}-i \kappa \mathrm{K}) j_{\varphi}=j_{\varphi}^{s}
$$

Here, $\kappa=h \sigma \mu \omega R$ is a system parameter, which represents the contribution of the inductive effects. A numerical approximation $j_{\varphi}^{(N)}$ of the true $j_{\varphi}$ is given by

$$
j_{\varphi}^{(N)}=\sum_{n=0}^{N} \frac{1}{1-i \kappa \lambda_{n}^{(N)}}\left(j_{\varphi}^{s}, u_{n}^{(N)}\right) u_{n}^{(N)},
$$

where $\lambda_{n}^{(N)}$ and $u_{n}^{(N)}$ are the eigenvalues and eigenfunctions, respectively, of the finite rank operator $\mathrm{K}^{(N)}$ that is used to replace $\mathrm{K}$. The series in (62) can also be written as

$$
j_{\varphi}^{(N)}=\sum_{n=0}^{N}\left[\frac{1}{1+\left(\kappa \lambda_{n}^{(N)}\right)^{2}}+i \frac{\kappa \lambda_{n}^{(N)}}{1+\left(\kappa \lambda_{n}^{(N)}\right)^{2}}\right]\left(j_{\varphi}^{s}, u_{n}^{(N)}\right) u_{n}^{(N)} .
$$

In order for the inductive effects (represented by the imaginary part) to dominate the resistive effects (represented by the real part), $\kappa \lambda_{n}^{(N)}$ must be greater than one.

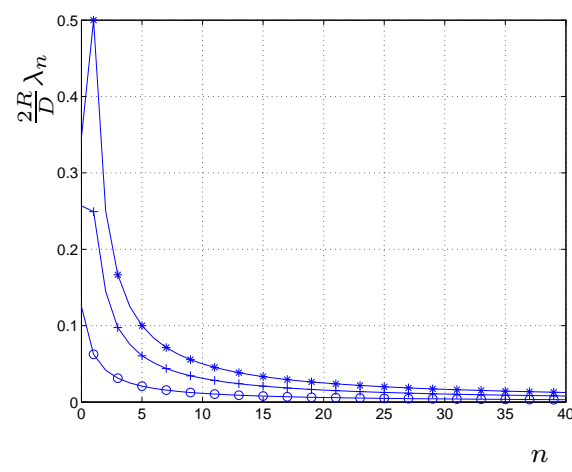

(a)

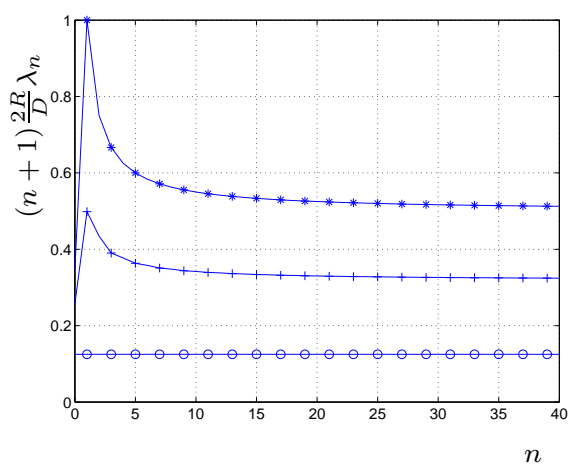

(b)

Figure 7: (a) The first 40 scaled eigenvalues of the operator $\mathrm{K}, 2 R \lambda_{n} / D(+)$, compared with a lower bound $1 /(8(n+1))(\circ)$, for $n \geq 0$, and an upper bound $1 /(2 n)(*)$, for $n \geq 1$ and $\log (2) / 2$ for $n=0$; (b) All values multiplied by $n+1$.

In [37], Reade has proved that the operator with kernel function $\log |z-\zeta|$ defined on $[-1,1]$ has negative eigenvalues $\mu_{n}$, which are bounded by

$$
-\pi \log 2 \leq \mu_{0} \leq-\frac{\pi}{4}, \quad-\frac{\pi}{n} \leq \mu_{n} \leq-\frac{\pi}{4(n+1)}, \quad n \geq 1 .
$$

We can approximate the eigenvalues of the operator $\mathrm{K}$, by using the matrix $\mathbf{A}$ from (41), which represents the finite rank operator $\mathrm{K}^{(N)}$. This means that we have to compute the eigenvalues of $\mathbf{G}^{-1} \mathbf{A}$, where the Gram matrix $\mathbf{G}$ is used to orthonormalize the basis functions. According 
to (34), the kernel is well approximated by the logarithmic function, multiplied by a factor $-1 /(2 \pi)$. Furthermore, due to scaling of the distances on radius $R$, the integral operator $\mathrm{K}$ is related to the integration interval $[-D /(2 R), D /(2 R)]$. We scale the eigenvalues $\lambda_{n}$, $n=0,1,2, \ldots$, of $\mathrm{K}$ with the factor $D /(2 R)$. Figures 7 (a) and (b) show that the eigenvalues are constrained by

$$
\frac{1}{8} \leq \frac{2 R}{D} \lambda_{0} \leq \frac{1}{2} \log 2, \quad \frac{1}{8(n+1)} \leq \frac{2 R}{D} \lambda_{n} \leq \frac{1}{2 n}, \quad n \geq 1 .
$$

Here, the upper and lower bounds are the same as in the case of an infinitely long plane rectangular strip; see [31].

The dominant eigenvalue $\lambda_{0}^{(N)}$ is equal to

$$
\lambda_{0}^{(N)}=0.257 \frac{D}{2 R} .
$$

The dependence of $\kappa$ on $R$ is not a characteristic feature of the system. But $\hat{\kappa}$, defined by

$$
\hat{\kappa}=\lambda_{0}^{(N)} \kappa=0.257 \frac{h \sigma \mu \omega D}{2}=0.807 h \sigma \mu f D,
$$

is a characteristic parameter of the system.

From (67), we see now that multiplying the width of the ring with a factor yields the same $\hat{\kappa}$ as multiplying the frequency with the same factor. This means that for a ring of $30 \mathrm{~cm}$ width instead of one that is 7.5 times narrower $(4 \mathrm{~cm})$, the frequency should be 7.5 times less than in the $4 \mathrm{~cm}$ case in order to find the same current distribution. In Figures 6 (c) and (d), the amplitude of the current and the phase-lag are shown for a ring of $30 \mathrm{~cm}$ width, with frequencies $f=13.3,53.3,93.3,113.3 \mathrm{~Hz}$. Comparing the results with the $4 \mathrm{~cm}$ case at the frequencies $f=100,400,700,1000 \mathrm{~Hz}$ - see Figures 4 (a) and (b) - we see that the distributions are indeed the same.

Next, we define the characteristic frequency

$$
f_{\text {char }}=\frac{1}{0.807 h \sigma \mu D}=\frac{1.234}{h \sigma \mu D}
$$

such that

$$
\hat{\kappa}=\frac{f}{f_{\text {char }}} .
$$

Then, $f=f_{\text {char }}$ represents the frequency at which prevailing resistive effects with respect to inductive effects change into prevailing inductive effects. This characteristic frequency can well be observed in the graphs of the resistance and the self-inductance against the frequency.

In Figure 8 (a), the resistance $\bar{R}$ of a $30 \mathrm{~cm}$ ring is shown for the frequency domain $f \in[0,10000] \mathrm{Hz}$. The resistance of the ring at $f=0 \mathrm{~Hz}$ is equal to

$$
\bar{R}_{D C}=\frac{2 \pi R}{h D \sigma} \approx 4.99 \cdot 10^{-5} \Omega
$$

The resistance increases with the frequency. In Figure 8 (b), the resistance is shown for frequencies up to $f=50 \mathrm{~Hz}$. We observe that at low frequencies the behavior of $\bar{R}$ is different from the one at high frequencies. The point of inflection in Figure 8 (b) is about $22 \mathrm{~Hz}$. This value corresponds very well to the theoretical value $f_{\text {char }}=22.3 \mathrm{~Hz}$, following from (68). 


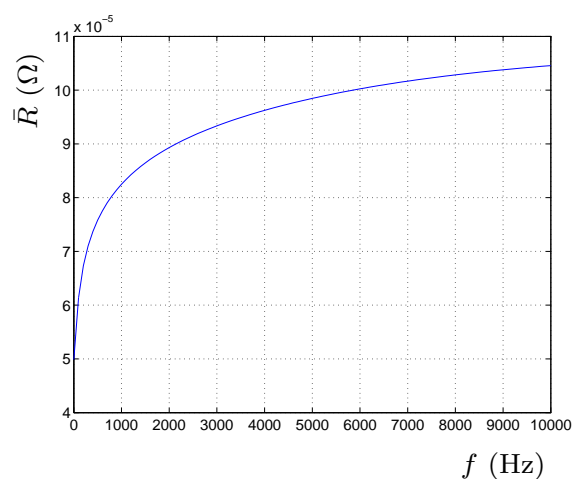

(a)

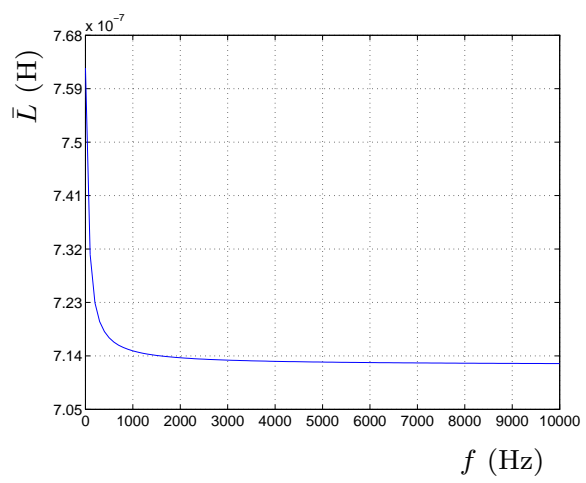

(c)

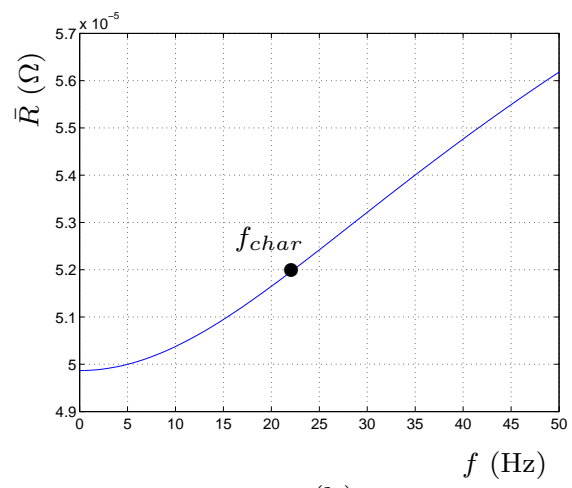

(b)

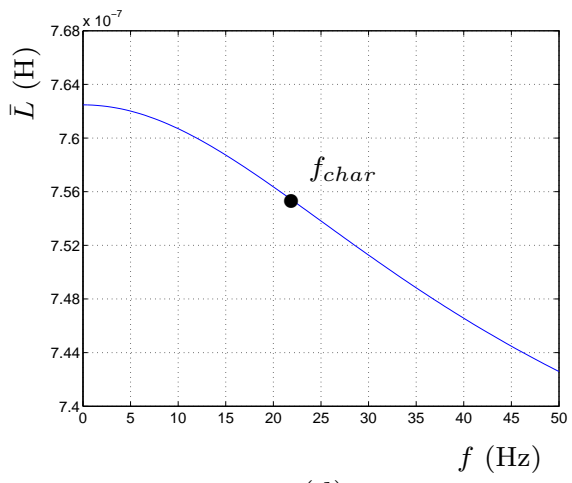

(d)

Figure 8: One ring of $30 \mathrm{~cm}$ width with a total current of $600 \mathrm{~A}$. (a) Resistance $\bar{R}$ at frequencies up to $f=10000 \mathrm{~Hz}$; (b) $\bar{R}$ at frequencies up to $f=50 \mathrm{~Hz}$. (c) Self-inductance $\bar{L}$ at frequencies up to $f=10000 \mathrm{~Hz}$; (d) $\bar{L}$ at frequencies up to $f=50 \mathrm{~Hz}$. 
Note that for a $4 \mathrm{~cm}$ ring the characteristic frequency is $f_{\text {char }}=167.0 \mathrm{~Hz}$, according to (68). Again, this value corresponds well to the point of inflection that can be observed in Figure 5.

For the self-inductance we find the same result: at the characteristic frequency $f_{\text {char }}=22.3$ $\mathrm{Hz}$, the behavior of $\bar{L}$ changes; see Figures 8 (c) and (d). Comparing this with the $4 \mathrm{~cm}$ case, we see again that the value is 7.5 times less.

\subsection{The Maxwell pair}

The second example we consider, is a special configuration of two rings. With a pair of rings we are able to create a gradient in the axial component of the magnetic field. In principle, a gradient coil is based on the properties of a so-called Maxwell pair. A Maxwell pair consists of two circular loops of wires, both of radius $R$, placed coaxial and parallel, and with the $z$-axis as central axis. If both wires are at equal distance from the origin and carry a current in anti-phase, then the $B_{z}$-field has a gradient in the origin. It has been proved that for a mutual distance between the wires equal to $R \sqrt{3}$, the field has no second, third and fourth order $z$-dependence; see Jin [46, Sect. 2.4]. In other words, at this distance the optimal linear field is achieved.

In the design of gradient coils, one uses a stream function approach, which results in a pattern of streamlines; see e.g. Peeren [22], [23], Tomasi [24]. For the manufacture of the coils, copper strips are placed on this pattern. For low frequencies and narrow strips, the currents cause a magnetic field that approximates the design field. However, increasing the frequencies or increasing the widths of the strips causes errors, which lead to unacceptable deviations in the gradient of the magnetic field.

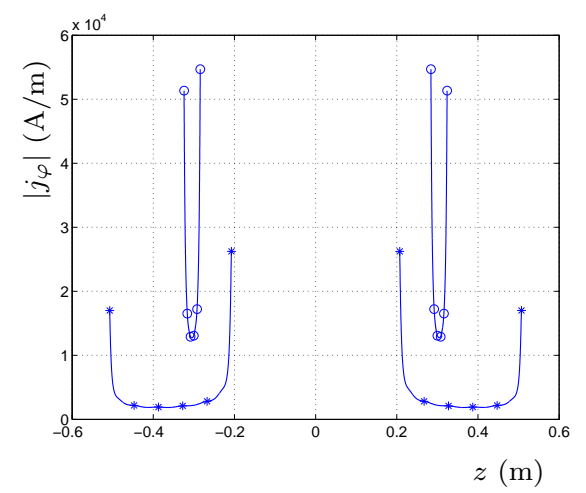

(a)

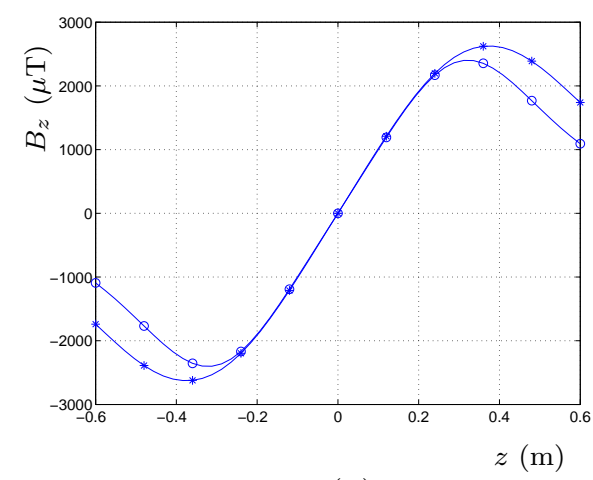

(b)

Figure 9: Two rings of $4 \mathrm{~cm}$ width (o) carrying 1526.0 $\mathrm{A}$ in anti-phase and two rings of $30 \mathrm{~cm}$ width $(*)$ carrying $1841.6 \mathrm{~A}$ in anti-phase, both at frequency $f=1000 \mathrm{~Hz}$. (a) Amplitude of the current distribution; (b) Magnetic field $B_{z}$ along the $z$-axis.

In this subsection we consider a pair of rings of $4 \mathrm{~cm}$ width and a pair of rings of 30 $\mathrm{cm}$ width. For a uniform distribution of the current in the rings (comparable with the DC situation) the distance between the rings for an optimal linearity of the $B_{z}$-field can be 
determined. We find an optimal distance equal to $60.8 \mathrm{~cm}$ for the $4 \mathrm{~cm}$ case and $71.4 \mathrm{~cm}$ for the $30 \mathrm{~cm}$ case. In order to obtain a desired gradient of $1.0 \cdot 10^{4} \mu \mathrm{T} / \mathrm{m}$, the current in the 4 $\mathrm{cm}$ rings must be $1526.0 \mathrm{~A}$ and in the $30 \mathrm{~cm}$ rings $1841.6 \mathrm{~A}$, and the currents in both rings are in anti-phase.

In Figures 9 (a) and (b), the amplitudes of the current distributions and the $z$-component of the magnetic field at the $z$-axis are shown for $f=1000 \mathrm{~Hz}$, for both cases. The gradient of the field is approximately $1.0 \cdot 10^{4} \mu \mathrm{T} / \mathrm{m}$ here, but this value is not exact. The difference, however, is not visible on the scale of this figure. A closer look on the difference is needed, which is presented in Figure 10.

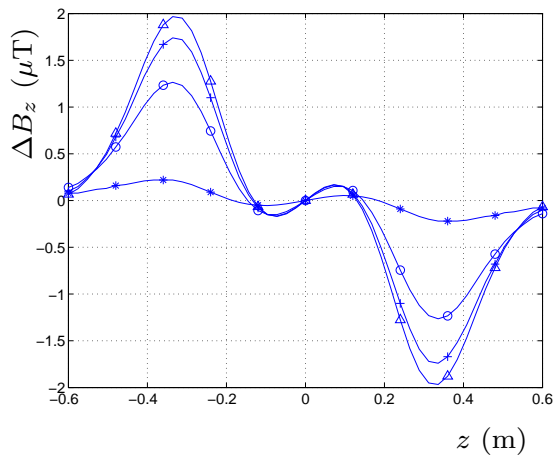

(a)

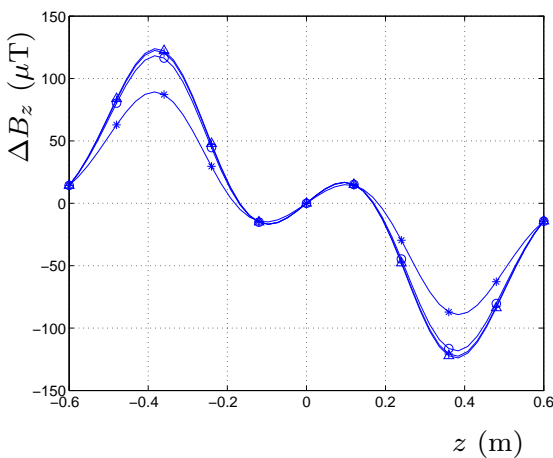

(b)

Figure 10: Difference between the $B_{z}$-field at $f=0 \mathrm{~Hz}$ and $f=100 \mathrm{~Hz}(*)$, $f=400 \mathrm{~Hz}(\circ), f=700 \mathrm{~Hz}(+), f=1000 \mathrm{~Hz}(\triangle)$. (a) Two rings of $4 \mathrm{~cm}$ width carrying $1526.0 \mathrm{~A}$ in anti-phase; (b) Two rings of $30 \mathrm{~cm}$ width carrying $1841.6 \mathrm{~A}$ in anti-phase.

In Figure 10 (a), the difference between the $B_{z}$-field at the $z$-axis at $f=0 \mathrm{~Hz}$ and at $f=100,400,700,1000 \mathrm{~Hz}$ is shown for the $4 \mathrm{~cm}$ case. We are especially interested in the deviations in the gradient at the origin. We observe that the error, i.e. the deviation from the ideal value of $1.0 \cdot 10^{4} \mu \mathrm{T} / \mathrm{m}$, increases with the frequency. For a frequency of $1000 \mathrm{~Hz}$ we observe an error in the gradient of approximately $3 \mu \mathrm{T} / \mathrm{m}$, meaning that the relative error is of the order $10^{-4}$. In Figure $10(\mathrm{~b})$, the difference between the $B_{z}$-field at the $z$-axis at $f=0 \mathrm{~Hz}$ and at $f=100,400,700,1000 \mathrm{~Hz}$ is shown for the $30 \mathrm{~cm}$ case. The relative error is in this case almost 10 times greater, i.e. of the order $10^{-3}$. Thus, the use of wider rings entails greater errors in the gradient of the field.

We see in Figure 10 how the eddy currents affect the magnetic field. Due to the edgeeffects, the gradient of $B_{z}$ at the origin has increased. In MRI, this deviation causes errors in the images. So, a time varying current in the rings gives not the same field as a DC current, for which the pair has been designed. This error can be compensated by the current supply. If we know the error of the field as a percentage of the desired field, the applied current should be decreased with the same frequency. Due to linearity of the system, the error in the gradient is then compensated. Note that this does not mean that higher-order effects are not amplified. 
In the error analysis, we only consider a small area around $z=0$. In a larger area, errors increase. We see that we cannot realize a linear magnetic field within a large region of interest with a Maxwell pair; more turns are needed. Another reason to use more turns is that the total dissipated power can be decreased and that a lower current needs to be supplied. In the next subsection we consider a realistic z-coil, modeled by 24 rings on the cylinder.

The resistance of a system of two rings is determined from (58), i.e. the total dissipated power divided by the squared effective current. In Figure 11 (a), the resistance $\bar{R}$ is shown for the $4 \mathrm{~cm}$ case for the frequency range $[0,10000] \mathrm{Hz}$. We observe that $\bar{R}$ increases with the frequency. The resistance $\bar{R}_{D C}$ at $f=0 \mathrm{~Hz}$ is verified analytically by taking double the circumferential length divided by the conductivity times the cross-sectional surface, i.e.

$$
\bar{R}_{D C}=\frac{4 \pi R}{h D \sigma} \approx 7.48 \cdot 10^{-4} \Omega .
$$

In Figure 11 (b), the resistance is shown for the smaller range of frequencies up to $f=400$ $\mathrm{Hz}$, with a higher resolution than used in the range from 0 to $10000 \mathrm{~Hz}$. We observe a point of inflection at the same characteristic frequency as in the example of one ring, i.e. $f_{\text {char }}=167.0$ Hz. This corresponds to the formula for $f_{\text {char }}$ in (68), in which the width of one ring is used.

The self-inductance of a system of two rings is determined from (60), i.e. the total magnetic energy divided by the squared effective current. In Figure 11 (c), the self-inductance $\bar{L}$ is shown for the $4 \mathrm{~cm}$ case for frequencies up to $10000 \mathrm{~Hz}$. We see that $\bar{L}$ decreases with the frequency. For low frequency range, from 0 to $400 \mathrm{~Hz}$, the self-inductance shows a point of inflection at $f_{\text {char }}=167.0 \mathrm{~Hz}$; see Figure $11(\mathrm{~d})$. The inflection is at the same characteristic frequency as found for the resistance and the example of one ring.

In Figure 12, the resistance and the self-inductance are shown for two rings of $30 \mathrm{~cm}$ width. We observe in both $\bar{R}$ and $\bar{L}$ a characteristic frequency $f_{\text {char }}=22.3 \mathrm{~Hz}$. This value corresponds to $f_{\text {char }}$ of one ring of $30 \mathrm{~cm}$ width and is verified analytically by (68). 


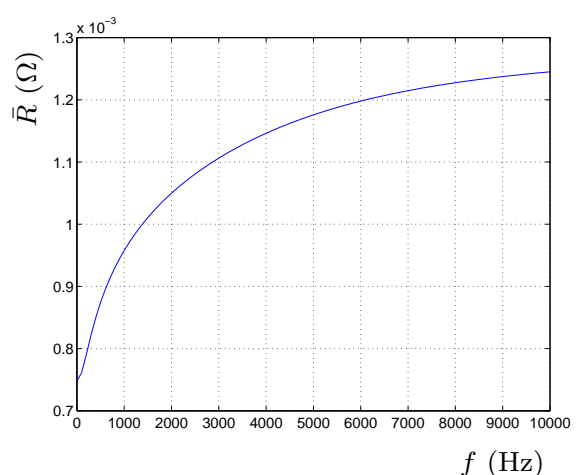

(a)

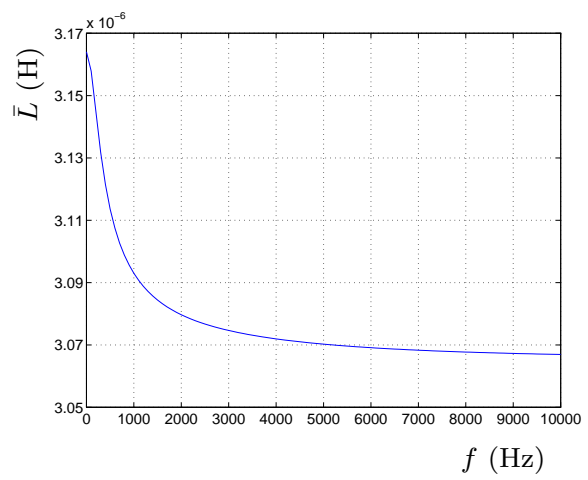

(c)

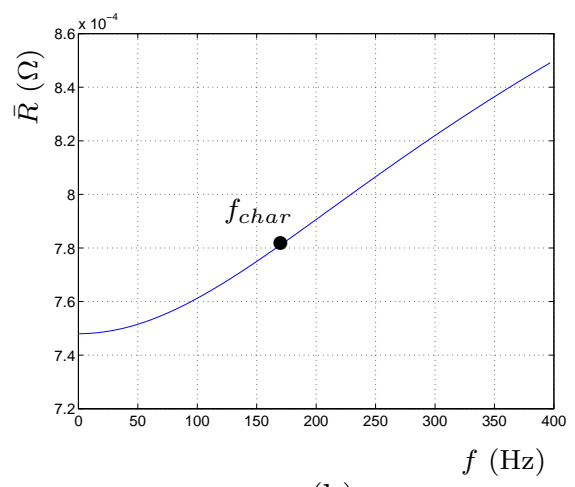

(b)

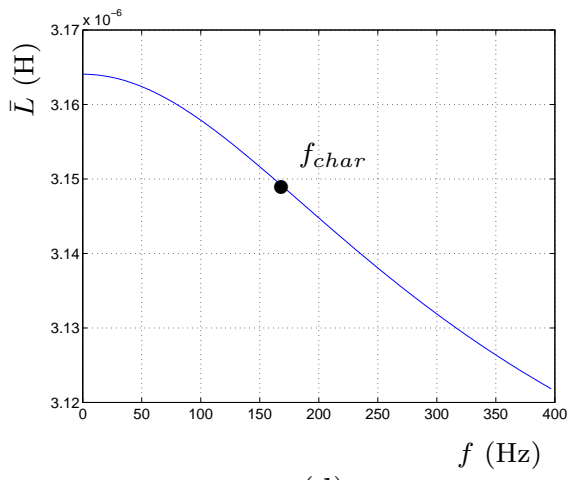

(d)

Figure 11: Two rings of $4 \mathrm{~cm}$ width carrying 1526.0 $\mathrm{A}$ in anti-phase. (a) Resistance $\bar{R}$ at frequencies up to $f=10000 \mathrm{~Hz}$; (b) $\bar{R}$ at frequencies up to $f=50 \mathrm{~Hz}$. (c) Self-inductance $\bar{L}$ at frequencies up to $f=10000 \mathrm{~Hz}$; (d) $\bar{L}$ at frequencies up to $f=50 \mathrm{~Hz}$. 


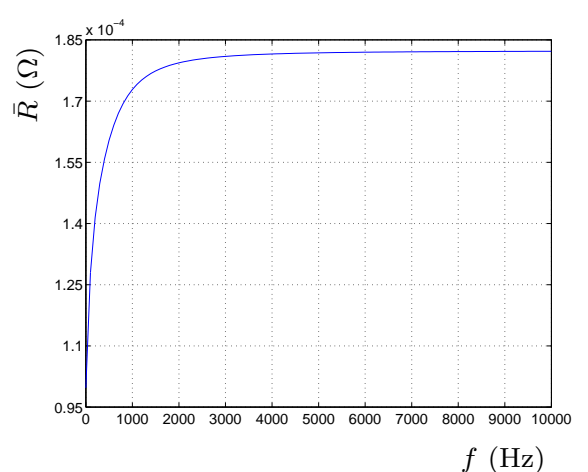

(a)

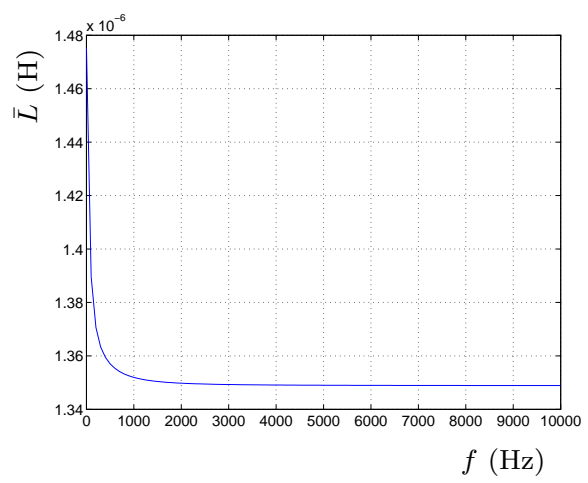

(c)

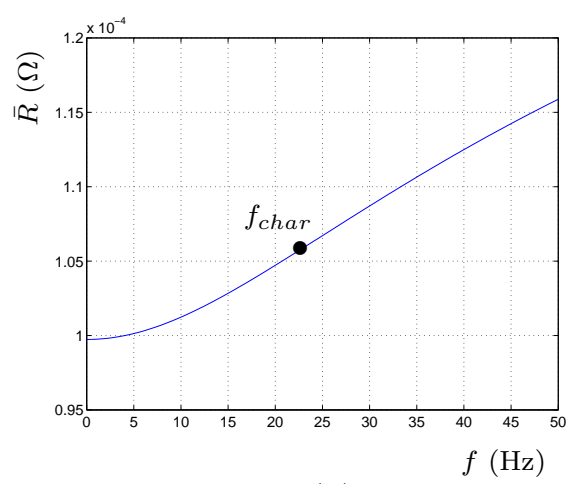

(b)

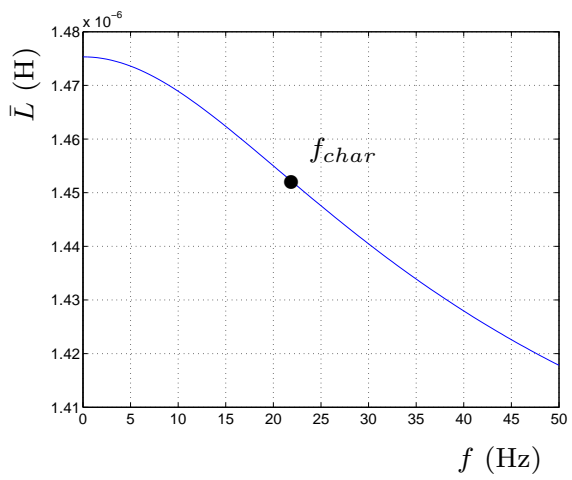

(d)

Figure 12: Two rings of $30 \mathrm{~cm}$ width carrying $1841.6 \mathrm{~A}$ in anti-phase. (a) Resistance $\bar{R}$ at frequencies up to $f=10000 \mathrm{~Hz}$; (b) $\bar{R}$ at frequencies up to $f=50 \mathrm{~Hz}$. (c) Self-inductance $\bar{L}$ at frequencies up to $f=10000 \mathrm{~Hz}$; (d) $\bar{L}$ at frequencies up to $f=50 \mathrm{~Hz}$. 


\subsection{A realistic $\mathrm{z}$-coil}

The third example we consider, is a set of 24 rings. This set describes a realistic z-coil. All 24 rings have a width of $2 \mathrm{~cm}$ and a radius of $35 \mathrm{~cm}$. The positions are determined by a gradient coil design program. In this program, the magnetic energy is minimized, subject to the requirements that the gradient of the magnetic field at the origin is $1.0 \cdot 10^{4} \mu \mathrm{T} / \mathrm{m}$, the second, third, and fourth order $z$-dependence are zero, and all currents are on the radius $R=0.35 \mathrm{~m}$. The design program determines a pattern of streamlines of the current. From this pattern a set of 24 rings is selected. We assign to each ring a width of $2 \mathrm{~cm}$ and compute again the field caused by the discretized currents. The error of the discretized current, in terms of the first- and third-order $z$-dependence, is compensated by scaling of the geometry. The resulting begin and end positions are listed in Table 2.

Table 2: Positions of rings and directions of currents in realistic z-coil.

\begin{tabular}{|c|cc|c||c|cc|c|}
\hline Ring & Left & Right & Dir. & Ring & Left & Right & Dir. \\
\hline 1 & -0.5277 & -0.5077 & -1 & 13 & 0.0306 & 0.0506 & -1 \\
\hline 2 & -0.4267 & -0.4067 & -1 & 14 & 0.0738 & 0.0938 & -1 \\
\hline 3 & -0.3753 & -0.3553 & -1 & 15 & 0.1572 & 0.1772 & 1 \\
\hline 4 & -0.3386 & -0.3186 & -1 & 16 & 0.1896 & 0.2096 & 1 \\
\hline 5 & -0.3090 & -0.2890 & -1 & 17 & 0.2151 & 0.2351 & 1 \\
\hline 6 & -0.2830 & -0.2630 & -1 & 18 & 0.2389 & 0.2589 & 1 \\
\hline 7 & -0.2589 & -0.2389 & -1 & 19 & 0.2630 & 0.2830 & 1 \\
\hline 8 & -0.2351 & -0.2151 & -1 & 20 & 0.2890 & 0.3090 & 1 \\
\hline 9 & -0.2096 & -0.1896 & -1 & 21 & 0.3186 & 0.3386 & 1 \\
\hline 10 & -0.1772 & -0.1572 & -1 & 22 & 0.3553 & 0.3753 & 1 \\
\hline 11 & -0.0938 & -0.0738 & 1 & 23 & 0.4067 & 0.4267 & 1 \\
\hline 12 & -0.0506 & -0.0306 & 1 & 24 & 0.5077 & 0.5277 & 1 \\
\hline
\end{tabular}

The total current in each ring is $180.52 \mathrm{~A}$, but not all currents are in phase. In Table 2, the phases are represented by the column called direction. A one stands for a current in phase and a minus one stands for a current in anti-phase. We see that the rings are not equally distributed and that the two halves do not exactly have opposite phase. This all has to do with the magnetic energy that is minimized.

In Figure 13, the amplitude of the current density is shown for an applied frequency of $1000 \mathrm{~Hz}$. The average current density in each ring is equal to

$$
j^{c}=\frac{\sum_{l=1}^{L} I_{l}}{\sum_{l=1}^{L} D_{l}}=9.03 \cdot 10^{3} \mathrm{~A} / \mathrm{m} .
$$

We observe local edge-effects in the rings as well as a global edge-effect in the whole system. We see how opposite currents amplify each other (e.g. in the two halves of the coil), whereas currents in the same direction damp each other (e.g. within one half of the coil).

In Figure 14, the magnetic field of the set of 24 rings is presented. The contourlines of the $B_{z}$ field in the $(y, z)$-plane are shown in Figure 14 (a). The frequency used is $f=1000$ $\mathrm{Hz}$. The linearity of the field is characterized by parallel contour lines. We observe a region 


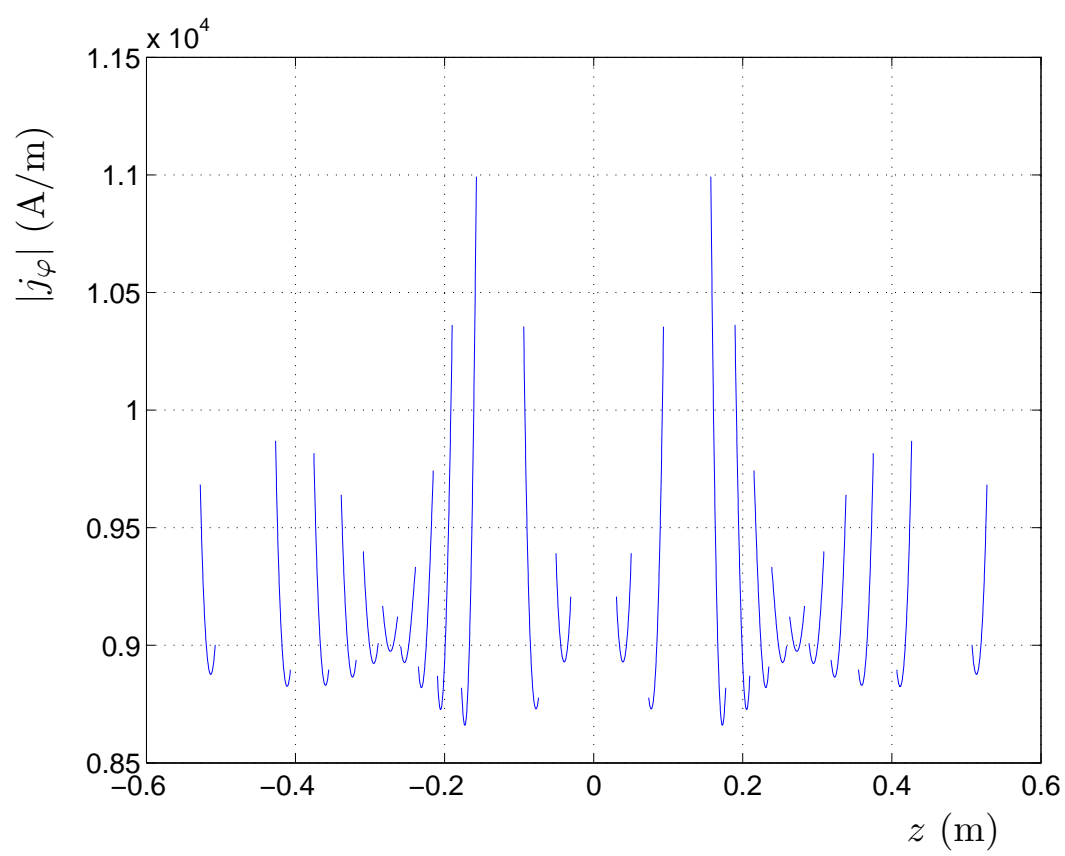

Figure 13: Amplitude of the current distribution in a set of 24 rings representing a z-coil. Each ring carries a current of $180.52 \mathrm{~A}$ at frequency $f=1000 \mathrm{~Hz}$.

around the origin in which the field is linear. In Figure 14 (b), the difference of the field with the DC field is presented by contour lines. This figure gives an indication of the third-order effects.

In Figure 14 (c), the magnetic field on the $z$-axis is shown, for the frequencies $f=$ $100,400,700,1000 \mathrm{~Hz}$. The gradient of the field is approximately $1.0 \cdot 10^{4} \mu \mathrm{T} / \mathrm{m}$, but not exact. The difference is not visible on the scale of the figure. For that, we consider the difference between the field caused by alternating currents and a direct current. In Figure 14 (d), the results are shown for the frequencies $f=100,400,700,1000 \mathrm{~Hz}$. The error of the gradient at $f=1000 \mathrm{~Hz}$ is approximately $0.5 \%$ of the desired gradient. Compensation of the gradient is possible when the applied current in the rings is increased by $0.5 \%$. Higher-order effects are still present.

The resistance of a system of 24 rings is determined from (58). In Figure 15 (a), the resistance $\bar{R}$ is shown for the frequency range $[0,10000] \mathrm{Hz}$. We observe that $\bar{R}$ increases with the frequency. The resistance $\bar{R}_{D C}$ at $f=0 \mathrm{~Hz}$ is verified analytically according to

$$
\bar{R}_{D C}=\frac{48 \pi R}{h D \sigma} \approx 1.79 \cdot 10^{-2} \Omega .
$$

In Figure 15 (b), $\bar{R}$ is shown for the smaller range of frequencies up to $f=800 \mathrm{~Hz}$, with a higher resolution than used in the range from 0 to $10000 \mathrm{~Hz}$. We observe a point of inflection at the characteristic frequency $f_{\text {char }}=334.0 \mathrm{~Hz}$. This corresponds to the formula for $f_{\text {char }}$ in (68), in which a width of $2 \mathrm{~cm}$ has to be substituted.

The self-inductance of a system of 24 rings is determined from (60). In Figure 15 (c), $\bar{L}$ 


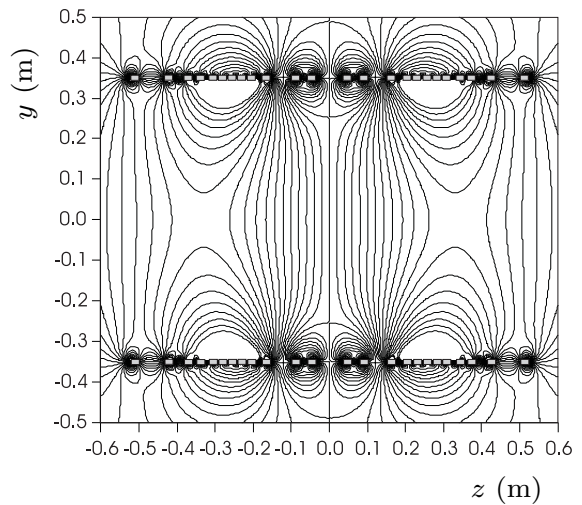

(a)

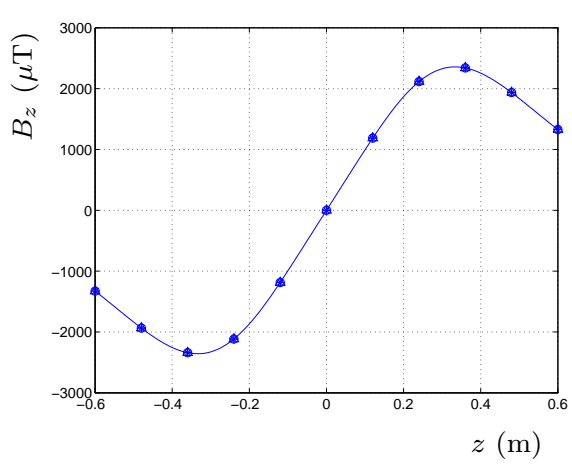

(c)

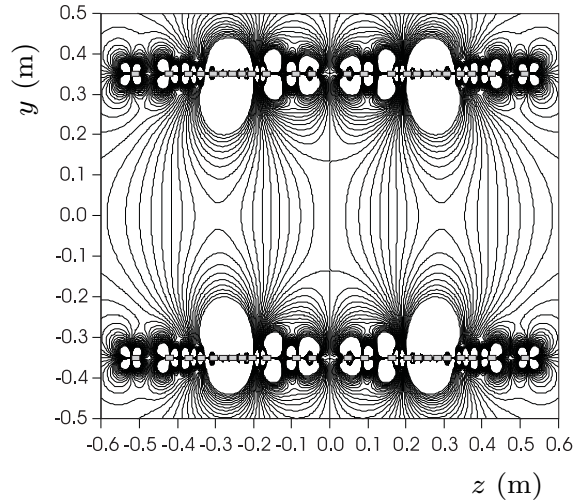

(b)

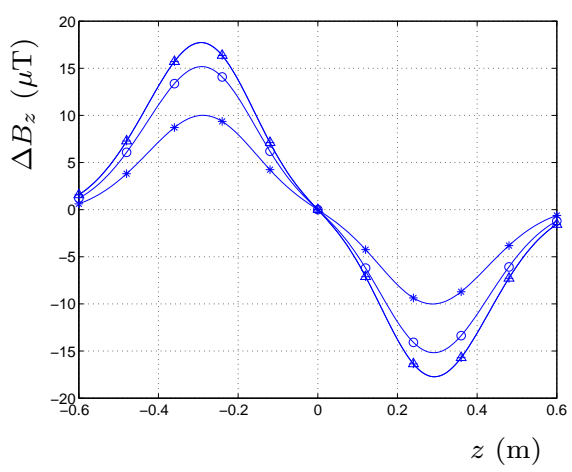

(d)

Figure 14: Magnetic field of a set of 24 rings representing a z-coil. (a) Contour lines $\mathrm{f} B_{z}$ at $f=1000 \mathrm{~Hz}$; (b) Contour lines of the difference between $B_{z}$ at $f=1000 \mathrm{~Hz}$ and $B_{z}$ at $f=0 \mathrm{~Hz}$; (c) $B_{z}$ on $z$-axis at $f=1000 \mathrm{~Hz}$; (d) Difference between $B_{z}$ at $f=100 \mathrm{~Hz}(*)$, $f=400 \mathrm{~Hz}(\circ), f=700 \mathrm{~Hz}(+), f=1000 \mathrm{~Hz}(\triangle)$ and $B_{z}$ at $f=0 \mathrm{~Hz}$ on $z$-axis. 
is shown for frequencies up to $10000 \mathrm{~Hz}$. The self-inductance decreases with the frequency. For low frequency range, from 0 to $800 \mathrm{~Hz}$, the self-inductance shows a point of inflection at $f_{\text {char }}=334.0 \mathrm{~Hz}$; see Figure $15(\mathrm{~d})$. The inflection is at the same characteristic frequency as found for the resistance.

The total dissipated power is less in a set of 24 rings than in a set of 2 rings. Namely, if we consider the Maxwell pair consisting of two rings of $4 \mathrm{~cm}$ width, then $\bar{R}$ and $\bar{L}$ can be obtained from Figure 11. At a frequency of $1000 \mathrm{~Hz}$ (for example), we find

$$
\bar{R}=9.6 \cdot 10^{-4} \Omega, \quad \bar{L}=3.09 \cdot 10^{-6} H .
$$

With a current $I=1526.0$ A, the time-averaged magnetic energy is determined from (60):

$$
\bar{U}_{m}=\frac{1}{2} \bar{L} I^{2}=3.60 \mathrm{~J} .
$$

The dissipated power follows from (58):

$$
\bar{P}_{\text {diss }}=I^{2} \bar{R}=2.24 \cdot 10^{3} \mathrm{~W} .
$$

For the set of 24 rings, we determine from Figure 15 that at $f=1000 \mathrm{~Hz}$

$$
\bar{R}=2.6 \cdot 10^{-2} \Omega, \quad \bar{L}=1.32 \cdot 10^{-4} \mathrm{H} .
$$

From this, we obtain for currents equal to $I=180.52 \mathrm{~A}$,

$$
\bar{U}_{m}=2.15 \mathrm{~J}, \quad \bar{P}_{\text {diss }}=8.47 \cdot 10^{2} \mathrm{~W} .
$$

Hence, the total dissipated power in a set of 24 rings is less than in 2 rings, while in both situations the same gradient in the magnetic field is created. The overall advantage is that the current that has to be supplied to the 24 rings is almost ten times less than the one needed in the 2 rings to create the same gradient.

\section{Conclusions and recommendations}

The most important conclusions from this research are the following:

- The current in the geometry of thin rings, which appear in the gradient coils of an MRIscanner, can be modeled as surface currents by averaging with respect to the thickness. The mathematical model is an integral equation of the second kind for the current density.

- The Galerkin method with global basis functions is the dedicated method for the considered configurations. Legendre polynomials are the most appropriate choice to describe the dependence of the current with respect to the transverse direction.

- The logarithmic part of the kernel describes the principal behavior of the currents. The contribution of the regular part of the kernel is small, in the order of one percent of the total. For designers, this is an acceptable degree of accuracy.

- The integral equation for a set of plane rectangular strips represents a model that describes qualitatively the current distribution and characteristic effects for ring-shaped configurations. 


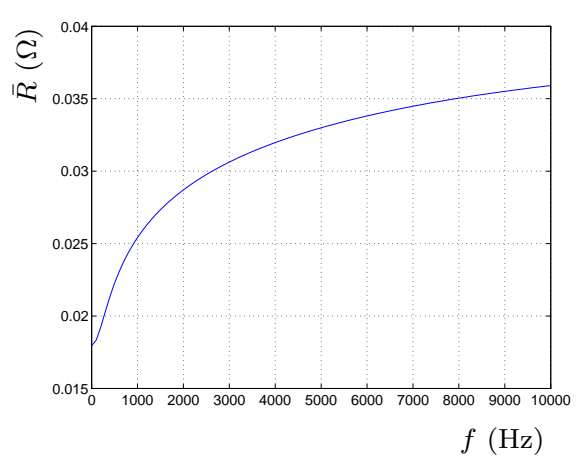

(a)

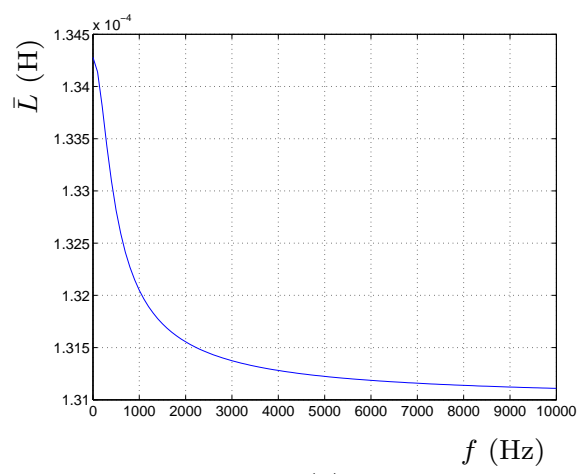

(c)

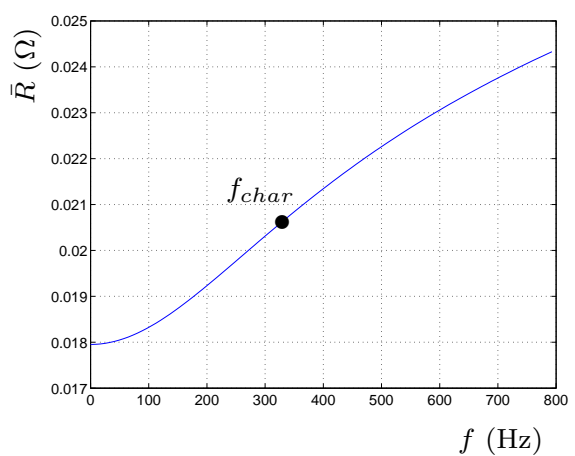

(b)

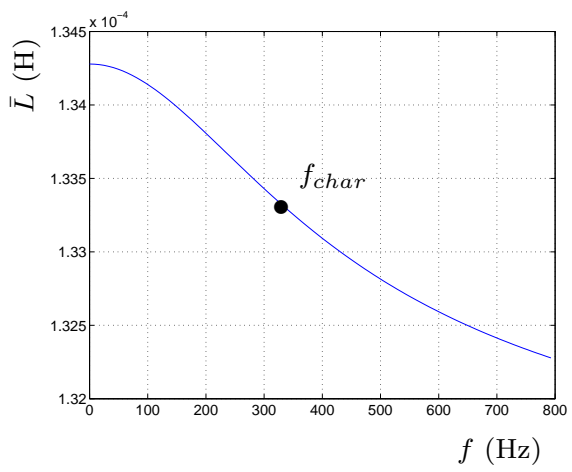

(d)

Figure 15: Resistance and self-inductance of a set of 24 rings representing a z-coil. (a) $\bar{R}$ at frequencies up to $f=10000 \mathrm{~Hz}$; (b) $\bar{R}$ at frequencies up to $f=400 \mathrm{~Hz}$; (c) $\bar{L}$ at frequencies up to $f=$ $10000 \mathrm{~Hz}$; (d) $\bar{L}$ at frequencies up to $f=400 \mathrm{~Hz}$. 
- The use of applied analysis, in particular explicit formulae for the special functions, makes our implementation very efficient from a computational point of view.

- Use of Legendre polynomials yields rapid convergence. For most of the computations, only eight Legendre polynomials are needed to obtain an accuracy of $99 \%$. For high frequencies (close to $10^{4} \mathrm{~Hz}$ ), we propose to use up to twelve polynomials.

- The method proposed in this paper provides a fast approximation of the current distribution. The implementation makes use of little memory, this in contrast to numerical packages, in which a lot of elements (memory) is needed to come to a high accuracy.

- Edge-effects become stronger when the frequency increases. Together with the phaselag, distortions in the magnetic field are explained. Consequently, the resistance of a system increases with the frequency, whereas the self-inductance decreases with the frequency.

For all simulations performed for the used examples, the computation times were in the order of one second (on a PC with an Intel Pentium 3 processor at $797 \mathrm{MHz}$, and $256 \mathrm{MB}$ of RAM). However, if one wants to simulate large sets of strips, we recommend the following modifications.

First, the Galerkin matrix $\mathbf{A}$ is dense, but diagonally dominant. We may replace $\mathbf{A}$ by $\mathbf{A}_{\tau}$, where $\mathbf{A}_{\tau}$ is obtained by dropping all elements smaller than $\tau$, i.e. $\left|A_{i j}\right|<\tau(\tau$ is often called a drop tolerance), thus eliminating much of the set-up time. The reason that the off-diagonal elements become smaller when they are further removed from the diagonal is that the distance between the two respective strips increases, resulting in smaller mutual inductions.

Second, the kernel function in the integral equation is logarithmically singular. Using only the logarithmic part, i.e. with the logarithmic function as kernel, we obtain the principal part of the solution. The resulting integration can be done fully analytically, leading to a simple formula. The remaining parts are regular and have been computed numerically. These computations regard numerical integrations, which become time consuming if the order of the basis functions is high. The regular parts have been computed for the completeness of the solution. However, if we neglect them, then the errors will be within one percent of the total solution. Moreover, the computation time will be reduced by more than ninety percent.

\section{References}

[1] C. N. Chen and D. I. Hoult. Biomedical magnetic resonance technology. Hilger, Bristol, 1989.

[2] M. T. Vlaardingerbroek and J. A. den Boer. Magnetic Resonance Imaging. SpringerVerlag, Berlin, 1999.

[3] J. A. Tegopoulos and M. Kriezis. Eddy currents in linear conducting media. Elsevier, Amsterdam, 1985.

[4] R. L. Stoll. The analysis of eddy currents. Clarendon Press, Oxford, 1974.

[5] J. Lammeraner and M. Stafl. Eddy currents. Hiffe books, London, 1966.

[6] P. C. Rem. Eddy current separation. Eburon, Delft, 1999. 
[7] E. Simeu and D. Georges. Modeling and control of an eddy current brake. Control Engineering Practice, 4:19-26, 1996.

[8] P. Sergeant, L. Dupré, J. Melkebeek, and L. Vandenbossche. Magnetic field computation for optimized shielding of induction heaters. Journal of Computational and Applied Mathematics, 168:437-446, 2004.

[9] D. W. Harberts. Dissipation and ringing of CRT deflection coils. PhD thesis Eindhoven University of Technology, Eindhoven, 2001.

[10] T. Teramoto, T. Nagai, Y. Utsugi, and M. Saito. Numerical analysis on fracture behavior of a single-edge-cracked plate subjected to electromagnetic force. Fusion Engineering and Design, 42:389-394, 1998.

[11] B. A. Hargreaves, C. H. Cunningham, D. G. Nishimura, and S. M. Conolly. Variable-rate selective excitation for rapid mri sequences. Magnetic Resonance in Medicine, 52:590597, 2004.

[12] U. D. Nguyen, J. S. Brown, I. A. Chang, J. Krycia, and M. S. Mirotznik. Numerical evaluation of heating of the human head due to magnetic resonance imaging. IEEE Transactions on Biomedical Engineering, 51:1301-1309, 2004.

[13] P. Mansfield, R. M. Bowley, and B. Haywood. Controlled e-field gradient coils. Magnetic Resonance Materials in Physics Biology and Medicine, 16:113-120, 2003.

[14] P. T. While and L. K. Forbes. Electromagnetic fields in the human body due to switched transverse gradient coils in mri. Physics in Medicine and Biology, 49:2779-2798, 2004.

[15] F. Romeo and D. I. Hoult. Magnetic field profiling: Analysis and correcting coil design. Magnetic Resonance in Medicine, 1:44-65, 1984.

[16] H. Suits and D. E. Wilken. Improving magnetic field gradient coils for nmr imaging. Journal of Physics E: Instrumentation, 22:565-573, 1989.

[17] E. Wong and A. Jesmanowicz. Coil optimization for mri by conjugate gradient descent. Magnetic Resonance in Medicine, 21:39-48, 1991.

[18] S. Crozier and D. M. Doddrell. Gradient coil design by simulated annealing. Journal of Magnetic Resonance, 103:354-357, 1993.

[19] T. A. Frenkiel, A. Jasinski, and P. G. Morris. Apparatus for generation of magnetic field gradient wave forms. Journal of Physics E: Scientific Instrumentation, 21:374-377, 1988.

[20] H. Siebold. Gradient field coils for $\mathrm{mr}$ imaging with high spectral purity. IEEE Transaction on Magnetics, 26:897-900, 1990.

[21] R. Turner. A target field approach for optimal coil design. Journal of physics D: Applied physics, 19:147-151, 1986.

$[22]$ G. N. Peeren. Stream function approach for determining optimal surface currents. PhD thesis, Eindhoven University of Technology, 2003. 
[23] G. N. Peeren. Stream function approach for determining optimal surface currents. Journal of Computational Physics, 191:305-321, 2003.

$[24]$ D. Tomasi. Stream function optimization for gradient coil design. Magnetic Resonance in Medicine, 45:505-512, 2001.

[25] R. Turner. Passive screening of switched magnetic field gradients. Journal of physics E: Scientific instrumentation, 19:876-879, 1986.

[26] R. Bowtell and P. Mansfield. Gradient coil design using active magnetic screening. Magnetic Resonance in Medicine, 17:15-21, 1998.

[27] P. Mansfield and B. Chapman. Multishield active magnetic screening of coil structures in nmr. Journal of marnetic resonance, 72:211-223, 1987.

[28] P. Mansfield, B. Haywood, and R. Coxon. Active acoustic control in gradient coils for mri. Magnetic Resonance in Medicine, 46:807-818, 2001.

[29] W. Li, C. K. Mechefske, C. Gazdzinski, and B. K. Rutt. Acoustic noise and prediction in a 4-t mri scanner. Concepts in Magnetic Resonance Part B - Magnetic Resonance Engineering, 21B:19-25, 2004.

[30] T. Ulicevic. Skin effect in a gradient coil. Master's thesis, Eindhoven, 2001.

[31] T. Ulicevic, J. M. B. Kroot, S. J. L. van Eijndhoven, and A. A. F. van de Ven. Current distribution in a parallel set of conducting strips. Accepted for publication in Journal of Engineering Mathematics.

[32] J. M. B. Kroot. Current distribution in a gradient coil, modeled as circular loops of strips. Master's thesis, Eindhoven, 2002.

[33] J. M. B. Kroot, S. J. L. van Eijndhoven, and A. A. F. van de Ven. Eddy currents in a gradient coil, modelled as circular loops of strips. In G. Benqing and X. Xiaowen, editors, 3rd international conference on computational electromagnetics and its applications, Beijing, China, November 2-5, pages 5-8. Chinese Institute of Electronics, 2004.

[34] J. M. C. Tas. Modelling and analyzing the z-gradient coil in an mri scanner. Master's thesis, Eindhoven University of Technology, 2005.

[35] K. E. Atkinson. A survey of numerical methods for the solution of Fredholm integral equations of the second kind. Society for Industrial and Applied Mathematics, Philadelphia, 1976.

[36] K. E. Atkinson. The numerical solution of integral equations of the second type. Cambridge University Press, Cambridge, 1997.

[37] J. B. Reade. Asymptotic behaviour of eigen-values of certain integral equations. Proceedings of the Edinburgh Mathematical Society, 22:137-144, 1979.

[38] A. Kovetz. The principles of electromagnetic theory. Cambridge University Press, Cambridge, 1990. 
[39] J. A. Stratton. Electromagnetic theory. Mc. Graw-Hill, London, 1941.

[40] L. Klinkenbusch. Theorie der sphärischen Absorberkammer und des mehrschaligen Kugelschirmes. Habilitationsschrift Ruhr-Universität Bochum, Bochum, 1996.

[41] S. H. J. A. Vossen. A two-wire antenna system for detecting objects in a homogeneous dielectric half space. PhD thesis, Eindhoven University of Technology, 2003.

[42] M. Abramowitz and I. A. Stegun. Handbook of Mathematical Functions with Formulas, Graphs and Mathematical Tables. Dover Publications, New York, 1968.

[43] G. N. Watson. A treatise on the theory of Bessel functions: tables of Bessel functions. Cambridge University Press, Cambridge, 1922.

[44] A. P. Prudnikov. Integrals and series. USSR Academy of Sciences, Moscow, 1986.

[45] P. F. Byrd and M. D. Friedman. Handbook of Elliptic Integrals for Engineers and Scientists. Springer-Verlag, Berlin, 1971.

[46] J. M. Jin. Electromagnetic analysis and design in magnetic resonance imaging. CRC Press, London, 1999.

[47] W. R. Smythe. Static and dynamic electricity. Mc. Graw-Hill, New York, 1968.

[48] T. Ulicevic, J. M. B. Kroot, S. J. L. van Eijndhoven, and A. A. F. van de Ven. Accepted for Journal of Engineering Mathematics.

[49] J. M. B. Kroot. Analysis of eddy currents in a gradient coil. PhD thesis, Eindhoven University of Technology, 2005. 